\title{
Development and Validation of a Mathematical Model to Describe Anti-Cancer Prodrug Activation by Antibody-Enzyme Conjugates
}

\author{
TRACHETTE L. JACKSON ${ }^{a, *}$, PETER D. SENTER ${ }^{b}$ and JAMES D. MURRAYc
}

${ }^{2}$ Institute for Mathematics and its Applications, University of Minnesota, 426 Lind Hall, 207 Church Street S.E., Minneapolis, MN 55455; 'bepartment of Applied Mathematics, University of Washington Box 352420, Seattle WA 98195-2420; ' Seattle Genetics, Inc., 2221526 th Avenue SE, Bothell, WA 98021

(Received 6 October 1998; In final form 1 April 1999)

\begin{abstract}
A mathematical model has been developed for a two-step approach to cancer chemotherapy involving the use of targeted monoclonal antibody-enzyme conjugates for the selective activation of anti-cancer prodrugs. Theoretical analysis and numerical simulation are used to characterize critical parameters for intratumoral and systemic drug generation. The model suggests that the most important pharmacokinetic and clinical parameters for increased drug production in the tumor are the rate of prodrug clearance from the blood and the initial injected dose of prodrug. The physiological parameters with the most influence are the prodrug and drug permeability. The ratio of tumor to blood drug generation can best be improved by increasing the conjugate clearance from the blood and decreasing the rate at which prodrug is converted to active drug. Predictions from this model concerning intratumoral prodrug and drug levels are validated by comparison with experimental data. Finally, the effects of certain barriers to chemotherapeutic treatments including vascular heterogeneity and radially outward convection are studied. If vascular heterogeneity alone is considered, the model predicts that the highest drug levels will occur in the most poorly vascularized sections of the tumor. However, when the effects of convection directed radially outward is considered, the highest drug levels are seen in the semi-well vascularized regions. This implies that the rapidly growing periphery of the tumor and the semi-necrotic tumor interior will receive the least amount of drug. These mathematical model predictions can lead to improved treatment protocols for this two step approach to cancer chemotherapy.
\end{abstract}

Keywords: Mathematical model, cancer chemotherapy, antibody-enzyme conjugates, prodrugs

${ }^{*}$ Corresponding author. 


\section{INTRODUCTION}

Considerable cancer research has been directed towards the development of selective targeting strategies to improve the efficacy of chemotherapy. One such new and promising approach employs monoclonal antibody-enzyme conjugates for the activation of anti-cancer prodrugs. In this two-step approach, a mAb-enzyme conjugate that specifically binds to antigens presented on the surfaces of tumor cells is administered and is allowed to localize in the tumor and clear from the systemic circulation before treatment with a relatively nontoxic anti-cancer prodrug. Upon contact with the targeted antibodyenzyme conjugate, the prodrug is converted into an active cytotoxic drug. The advantages of this targeting strategy over that of direct antibody-drug conjugates [Pietersz et al., 1994] include the ability of the conjugate to catalytically generate large amounts of active drug. The drug thus formed can penetrate into regions of the tumor mass that are inaccessible to the conjugate. Pharmacokinetics studies have demonstrated that mAb-enzyme/prodrug combinations can result in high intratumoral drug concentrations [Bosslet et al., 1994], [Svensson et al., 1995], [Wallace et al., 1994], pronounced anti-tumor activities in an array of pre-clinical tumor models [Siemers et al., 1997], [Springer et al., 1991], [Meyer et al., 1993], [Eccles et al., 1994], [Kerr et al., 1995] and activities in the clinic [Bagshawe and Begent, 1996].

A number of factors can profoundly influence the therapeutic effects obtained from $\mathrm{mAb}$-enzyme/prodrug combinations. Solid tumors have complex geometric and physiologic properties which can cause high molecular weight agents, such as mAb-enzyme conjugates, to distribute in nonuniform manners. Vascular heterogeneity is an example of one such barrier to macromolecular drug delivery. The distance between capillary centers can vary widely in a tumor with most vessels located on the periphery [Endrich et al., 1979]. For this reason, mAbs used for cancer therapy tend to accumulate near well vascularized sections of the tumor with significantly reduced levels reaching the tumor interior.
Another barrier to intratumoral mAb-enzyme conjugate uptake results from the increased interstitial pressure inherent in the interior of tumor [Baxter and Jain, 1989]. The pressure is highest in the center of the tumor decreasing to normal levels in a thin boundary layer at the tumor periphery. The effect of this increased interstitial pressure is to drive a convective current radially outward. The convective flux acts to impede the diffusion of macromolecules in the interior of the tumor which can lead to nonuniform conjugate distribution.

Aside from the barriers to $\mathrm{mAb}$-enzyme penetration, factors such as enzyme kinetics, conjugate biodistribution, binding affinity, and conjugate and prodrug doses and dosing schedule can all affect therapeutic efficacy. Because of the degree of complexity inherent in this approach, several studies have been directed towards the development of mathematical models to predict the effects of these parameters and others on the intratumoral and systemic conjugate and drug concentrations [Baxter and Jain, 1996, Baxter et al., 1992]. In previous papers, we studied in detail the first step of this twostep approach to chemotherapy. In Jackson et al, 1999a, we present a mathematical model based on the biology of human 3677 melanoma xenografts in nude mice in order to determine the biodistribution, pharmacokinetics, and intratumoral localization properties of L49- $\beta$-lactamase fusion proteins in solid tumor masses. Analytic expressions for the total concentration of conjugate in the tumor, the time at which the concentration is maximal, and the half life of conjugate in the tissue were derived. The antibody-antigen dissociation ratio, the conjugate permeability, and the inter-capillary half distance within the tumor mass were found to strongly influence conjugate localization and retention in the tumor.

Here, we extend our previously described model for the prediction of intratumoral conjugate uptake to include the activation of a systemically administered prodrug. The goal in modeling conjugate distribution and drug generation is to determine how intratumoral drug concentrations can be maximized and how the targeting index (TI) which measures 
the ratio of drug generation in the tumor to that in the blood can be improved. The model we describe takes into account many parameters that were not previously considered, such as the effects of free and bound intratumoral conjugate activation of prodrug, the trafficking of drug into and out of cells, vascular heterogeneity, and outward convection currents. The model has been shown to accurately predict drug levels within tumors of animals receiving mAb-enzyme/prodrug combinations. This provides a means for determining optimal dosing strategies for treatment, and suggests future experimental directions.

\section{MODEL DEVELOPMENT AND ANALYSIS}

The two-step drug targeting strategy involving the combination of mAb-enzyme conjugates and a prodrug lends itself well to a three-compartment model formulation describing the tumor, plasma, and the normal tissues. The tumor compartment is then further subdivided into intracellular and extracellular space.

\section{Compartmental Approach}

The ' armacokinetics outside the tumor was modele by two well-mixed compartments, the blood compartment and the peripheral compartment representing all other non-tumor, non-blood tissues. The volume of the blood compartment was taken to be $2.5 \mathrm{ml}$, which is the average volume of blood in an $18-22 \mathrm{~g}$ nude mouse. The conjugate and prodrug are injected directly into the blood and distribute throughout the body via reversible transfer between the blood and normal tissues. Only from the blood can the conjugate and prodrug pass through the microvessel wall and access the tumor. The tumor was considered to contain both intracellular and extracellular space. Once in the tumor compartment, the conjugate can diffuse and bind to antigens presented on cell surfaces. Once bound, the majority of the conjugate remains of the cell surface and is not internalized. The prodrug is converted to active drug in the presence of the enzyme, the drug thus formed is internalized into cells or is cleared (See Figure 1).

\section{Tumor Geometry}

The structure of the tumor tissue was based on the conception of the tumor as equivalent to a collection of (Krogh) cylinders of tissue, each nourished by a single capillary, the collection possessing the same structure as a box of pencils [Krogh, 1919]. Each Krogh cylinder was considered to have an outer radius $L$ equal to half the average distance between capillary centers and an inner radius $\rho$ equal to the average radius of the tumor capillaries.

This geometrical description of the tumor together with the compartment approach was employed to describe the rate of change of the concentration of free and bound conjugate, prodrug, and drug in the tumor, blood, and peripheral compartments. The variables that were tracked are listed in Table I while the parameters that govern their rates of change are in Table II together with detailed references from which they were obtained.

\section{Model Equations}

In the microvessel, the concentration of conjugate, $c_{1}$, prodrug, $p_{1}$, and drug, $d_{1}$, satisfy

$$
\begin{aligned}
\frac{d c_{1}}{d t} & =-k_{12} c_{1}+k_{21} c_{2}-k_{e} c_{1} \\
& -\frac{2 P_{C} \rho}{L^{2}-\rho^{2}}\left(c_{1}-C_{F}(\rho, t)\right) \\
\frac{d p_{1}}{d t} & =-\bar{\kappa}_{12} p_{1}+\bar{\kappa}_{21} p_{2}-\bar{\kappa}_{\epsilon} p_{1} \\
& -\frac{2 P_{P} \rho}{L^{2}-\rho^{2}}\left(p_{1}-P(\rho, t)\right)-\frac{V_{\max } c_{1} p_{1}}{K_{m}+p_{1}} \\
\frac{d d_{1}}{d t} & =-\tilde{\kappa}_{12} d_{1}+\tilde{\kappa}_{21} d_{2}-\tilde{\kappa}_{\epsilon} d t \\
- & \frac{2 P_{D} \rho}{L^{2}-\rho^{2}}\left(d_{1}-D_{E}(\rho, t)\right)+\frac{V_{\max } c_{1} p_{1}}{K_{m}+p_{1}}
\end{aligned}
$$

where $k_{i j}, \bar{\kappa}_{i j}$, and $\tilde{\kappa}_{i j}$ are the experimentally determined compartmental transfer coefficients for the 

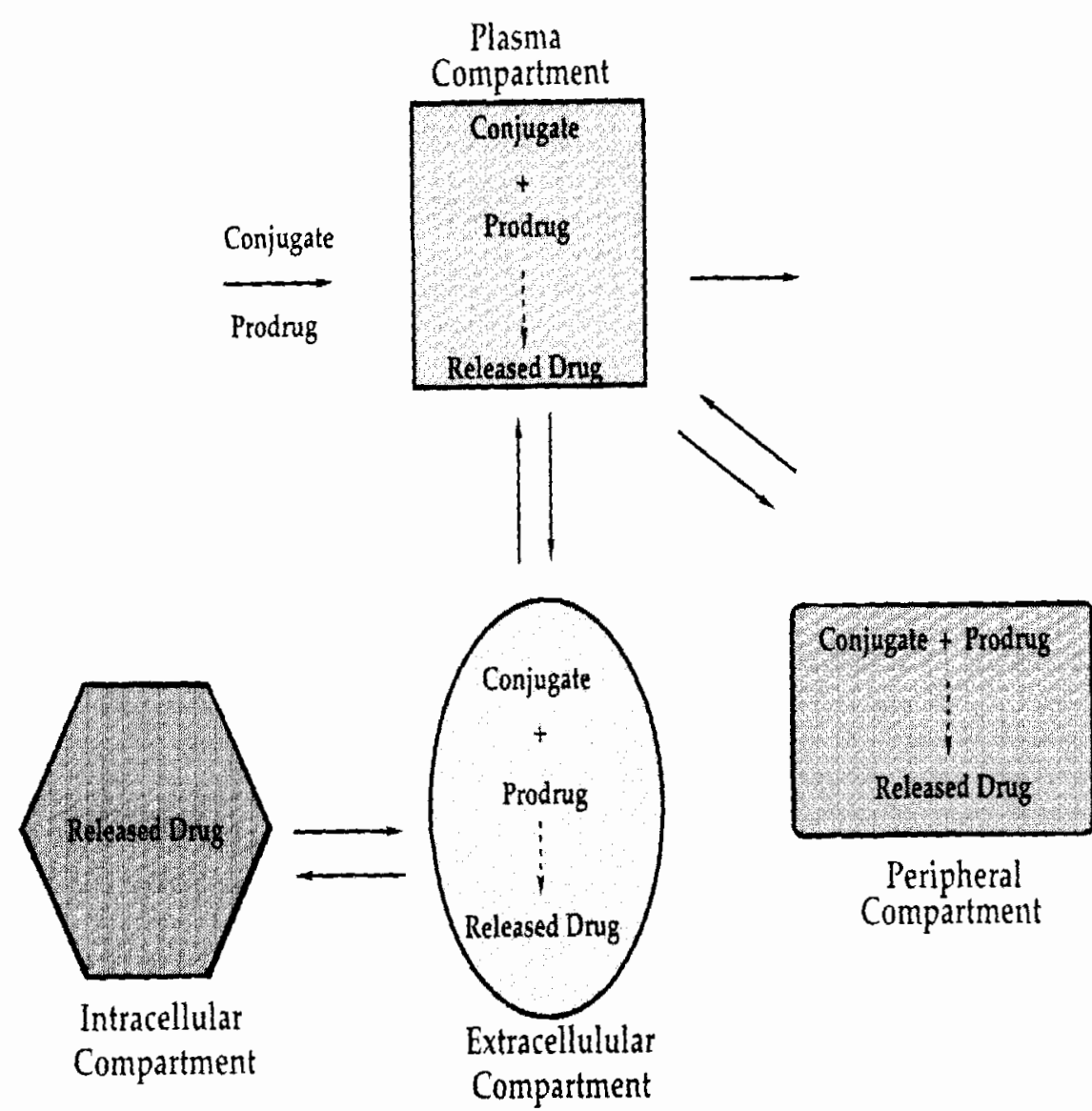

FIGURE 1 Diagram of the compartmental approach taken to model the activation of anti-cancer prodrug as a two-step chemotherapeutic strategy.

TABLE I Definition of the model variables and their dimensions

\begin{tabular}{ll}
\hline Variable & Definition \\
\hline$C_{F}(r, t)$ & Concentration of free conjugate in the tumor $(\mathrm{M})$ \\
$C_{B}(r, t)$ & Concentration of bound conjugate in the tumor $(\mathrm{M})$ \\
$P(r, t)$ & Concentration of prodrug in the tumor $(\mathrm{M})$ \\
$D_{E}(r, t)$ & Extracellular concentration of drug in the tumor $(\mathrm{M})$ \\
$D_{1}(r, t)$ & Intracellular concentration of drug in the tumor $(\mathrm{M})$ \\
$c_{1}(t)$ & Concentration of conjugate in the blood $(\mathrm{M})$ \\
$p_{1}(t)$ & Concentration of prodrug in the blood $(\mathrm{M})$ \\
$d_{1}(t)$ & Concentration of drug in the blood $(\mathrm{M})$ \\
$c_{2}(t)$ & Concentration of conjugate in the peripheral compartment $(\mathrm{M})$ \\
$p_{2}(t)$ & Concentration of prodrug in the peripheral compartment $(\mathrm{M})$ \\
$d_{2}(t)$ & Concentration of drug in the peripheral compartment $(\mathrm{M})$ \\
\hline
\end{tabular}

conjugate, prodrug, and drug respectively, and $k_{e}$, $\bar{\kappa}_{e}$, and $\widetilde{\kappa}_{e}$ are the blood elimination rates for each agent. The rate of transfer from microvessel to tumor is give by the product of the permeability and the surface area of the vessel divided by the volume of the cylinder. The prodrug conversion is reasonably taken to follow Michaelis-Menton kinetics with reaction parameters represented by $V_{\max }$ and $K_{M}$. 
TABLE II Definitions of model parameters and their units

\begin{tabular}{|c|c|}
\hline Parameter & Definition \\
\hline$C_{0}$ & Initial concentration of conjugate $(\mathrm{M})$ \\
\hline$P_{0}$ & Initial concentration of prodrug $(M)$ \\
\hline$k_{12}, k_{21}$ & $\begin{array}{l}\text { Conjugate compartmental transfer } \\
\text { coefficients }\left(\mathrm{s}^{-1}\right)\end{array}$ \\
\hline$k_{e}$ & Conjugate blood elimination rate $\left(\mathrm{s}^{-1}\right)$ \\
\hline $\bar{\kappa}_{12}, \bar{\kappa}_{21}, \tilde{\kappa}_{21}, \tilde{\kappa}_{21}$ & $\begin{array}{l}\text { Prodrug and drug compartmental transfer } \\
\text { coefficients }\left(s^{-1}\right)\end{array}$ \\
\hline $\bar{k}_{e}, \tilde{k}_{e}$ & $\begin{array}{l}\text { Prodrug and drug blood elimination } \\
\text { rate }\left(\mathrm{s}^{-1}\right)\end{array}$ \\
\hline$L$ & Krogh cylinder radius (mm) \\
\hline$\rho$ & Microvessel radius (mm) \\
\hline$P_{i}$ & $\begin{array}{l}\text { Permeability coefficient of the } \\
\text { microvessel wall }\left(\mathrm{mm} \mathrm{s}^{-1}\right)\end{array}$ \\
\hline$D_{i}$ & $\begin{array}{l}\text { Diffusion coefficient of agent, } i \text {, in tumor } \\
\text { tissue }\left(\mathrm{mm}^{2} \mathrm{~s}^{-1}\right)\end{array}$ \\
\hline$k_{q}, k_{d}$ & $\begin{array}{l}\text { Association and dissociation rate } \\
\qquad\left(\mathrm{M}^{-1} \mathrm{~s}^{-1}, \mathrm{~s}^{-1}\right)\end{array}$ \\
\hline$A_{0}$ & Antigen density $(\mathrm{M})$ \\
\hline$V_{\max }, K_{m i}$ & $\begin{array}{l}\text { Enzyme/Prodrug reaction kinetic } \\
\text { parameters }\left(\mathrm{s}^{-1}, \mathrm{M}\right)\end{array}$ \\
\hline$a_{I}, b_{I}$ & $\begin{array}{l}\text { Drug internalization rate } \\
\text { constants }\left(s^{-1}\right)\end{array}$ \\
\hline
\end{tabular}

In the peripheral compartment, which represents the normal tissues of the body, the concentration of conjugate, $c_{2}$, prodrug, $p_{2}$, and drug, $d_{2}$, satisfy

$$
\begin{aligned}
& \frac{d c_{2}}{d t}=k_{12} c_{1}-k_{21} c_{2} \\
& \frac{d p_{2}}{d t}=\bar{\kappa}_{12} p_{1}-\bar{\kappa}_{21} p_{2}-\frac{V_{\max } c_{2} p_{2}}{K_{m}+p_{1}} \\
& \frac{d d_{2}}{d t}=\tilde{\kappa}_{12} d_{1}-\tilde{\kappa}_{21} d_{2}+\frac{V_{\max } c_{2} p_{2}}{K_{m}+p_{1}}
\end{aligned}
$$

In the tumor, the extracellular concentrations of free conjugate, $C_{F}$; bound conjugate, $C_{B}$; prodrug, $P$; and drug, $D_{E}$, are governed by

$$
\begin{aligned}
& \frac{\partial C_{F}}{\partial t}=D_{C} \Delta C_{F}-k_{a} C_{F}\left(A_{0}-C_{B}\right)+k_{d} C_{B} \\
& \frac{\partial C_{B}}{\partial t}=k_{a} C_{F}\left(A_{0}-C_{B}\right)-k_{d} C_{B} \\
& \frac{\partial P}{\partial t}=D_{P} \Delta P-\frac{V_{\max }\left(C_{F}+C_{B}\right) P}{K_{m}+P} \\
& \frac{\partial D_{E}}{\partial t}=D_{D} \Delta D_{E}+\frac{V_{\max }\left(C_{F}+C_{B}\right) P}{K_{m}+P}-a_{I} D_{E}
\end{aligned}
$$

where $\Delta$ is the axially symmetric Laplacian operator, $k_{a}$ and $k_{d}$ are the experimentally determined antibody-antigen association and dissociation rates respectively; and $A_{0}$ is the initial antigen concentration in the tumor. The diffusion coefficient of each agent is given by $D_{i}, i=C, P, D$ and the cellular internalization rate is represented by the parameter $a_{I}$. The intracellular drug concentration, $D_{I}$ satisfies

$$
\frac{\partial D_{I}}{\partial t}=a_{I} D_{E}-b_{I} D_{I}
$$

where $b_{I}$ is included to account for any escape of the drug from inside the tumor cells.

The initial concentration of the antibody-enzyme conjugate, prodrug, and drug in each compartment must be given and boundary conditions governing the behavior at the boundaries must be prescribed to complete the model. Initially, the concentration of both free and bound conjugate, prodrug, and drug in the normal tissues and in the tumor is zero:

$$
\begin{array}{lll}
c_{2}(0)=0 & p_{2}(0)=0 & d_{2}(0)=0 \\
C_{F}(r, 0)=0 & C_{B}(r, 0)=0 & P(r, 0)=0 \\
D_{E}(r, 0)=0 & D_{I}(r, 0)=0
\end{array}
$$

The initial concentration of conjugate and prodrug in the blood compartment is the administered dose, $C_{0}$ and $P_{0}$, respectively. There is no drug in the blood initially.

$$
c_{1}(0)=C_{0} \quad p_{1}(\tau)=P_{0} \quad d_{1}(0)=0 .
$$

Here, $\tau$ is the time of prodrug administration. For this study, the baseline value for $\tau$ is $15 \mathrm{~h}$ after the injection of the antibody-enzyme conjugate.

Due to symmetry with surrounding Krogh cylinders, the net flux of all agents across the boundary of the tumor tissue, $r=L$, is zero:

$$
\frac{\partial C_{F}}{\partial r}(L, t)=0 \quad \frac{\partial P}{\partial r}(L, t)=0 \quad \frac{\partial D_{E}}{\partial r}(L, t)=0
$$

The flux across the blood vessel wall, $r=\rho$, is driven by the concentration difference in the two compartments and is mediated by the wall permeability.

$$
\frac{\partial C_{F}}{\partial r}(\rho, t)=-\frac{P_{C}}{D_{C}}\left[c_{1}(t)-C_{F}(\rho, t)\right]
$$




$$
\begin{aligned}
\frac{\partial P}{\partial r}(\rho, t) & =-\frac{P_{P}}{D_{P}}\left[p_{1}(t)-P(\rho, t)\right] \\
\frac{\partial D_{E}}{\partial r}(\rho, t) & =-\frac{P_{D}}{D_{D}}\left[d_{1}(t)-D_{E}(\rho, t)\right]
\end{aligned}
$$

\section{Data Analysis}

By incorporation of the experimental data, a leastsquares fit for the blood clearance of the antibodyenzyme conjugate, L49-sFv-bL, [Siemers et al., $1997]$ into the biexponential function $C=A e^{-\alpha t}+$ $B e^{-\beta t}$ was obtained, and values for $A, B, \alpha, \beta$ were determined. These values were in turn used to compute the compartmental transfer coefficients $k_{12}, k_{21}$, and $k_{\varepsilon}$. The diffusion coefficient, $D_{i}, i=$ $C, P, D$, was assumed to depend on the molecular weight, $M_{W}$ as $a\left(M_{W}\right)^{b}$ using published values for $a$ and $b$ [Nugent and Jain, 1984]. To compute the antigen density, $A_{0}$, the average value of the number of molecules per cell when saturated was $2.1 \times 10^{4}$ (molecules/cell). The cell density was estimated as $2.83 \times 10^{11}$ (cell/liter) [Baxter and Jain, 1991a] by assuming $50 \%$ cell fraction and an average cancer cell diameter of $15 \mu \mathrm{m}$ (cell density $=\left(\right.$ cell fraction $\left./(4 / 3) \pi r^{3}\right)$. Together. these values lead to the antigen density listed in Table III. The average distance between capillary centers was calculated as $J=\sqrt{(0.25 / \pi N)}$ where $N$ is the average number of capillaries in every $0.25 \mathrm{~mm}^{2}$ of tissue which was determined experimentally [Jackson et al., 1999b].

The alpha-decay rates for the blood clearance of the prodrug and drug were computed from the $\alpha$ half life data given in [Svensson et al., 1995] and [Robert et al., 1982] respectively. The rate of cellular internalization was estimated based on the experimentally determined value for cellular permeability of doxorubicin [Dordal et al., 1992]. They estimated the permeability coefficient of doxorubicin for two different cell lines to be $P_{c}=7.4 \times 10^{-5} \mathrm{~cm} / \mathrm{s}$. The parameter $\alpha_{I}$ was estimated from this result as

$$
a_{i}=\frac{P_{c}}{\text { cell density } \times \text { cylinder volume } \times \text { circumterence/cell }} .
$$

\begin{tabular}{|c|c|c|}
\hline Parameter & Value & Reference \\
\hline$L$ & $\begin{array}{l}0.11 \mathrm{~mm} \\
0.002 \mathrm{~mm}\end{array}$ & $\begin{array}{l}\text { [Jackson et al. 1998b] } \\
\text { [Jackson et al. 1998b] }\end{array}$ \\
\hline $\begin{array}{l}\rho \\
C_{0}\end{array}$ & $\begin{array}{l}0.002 \mathrm{~mm} \\
12 \times 10^{-7} \mathrm{M}\end{array}$ & $\begin{array}{l}\text { [Jackson et al. } \\
\text { [Siemers ef al. 1997] }\end{array}$ \\
\hline $\begin{array}{l}C_{0} \\
P_{0}\end{array}$ & $\begin{array}{l}1.2 \times 10^{-1} \mathrm{M} \\
6.4 \times 10^{-4} \mathrm{M}\end{array}$ & $\begin{array}{l}\text { [Siemers ef al., } 1997]^{\mathrm{A}} \\
{\text { [Current work }]^{\mathrm{f}}}^{\text {C. }}\end{array}$ \\
\hline $\begin{array}{l}k_{12} \\
k_{21} \\
k_{e} \\
\bar{k}_{t:} \\
\tilde{k}_{e}\end{array}$ & $\begin{array}{l}2.4 \times 10^{-5} \mathrm{~s}^{-1} \\
8.0 \times 10^{-5} \mathrm{~s}^{-1} \\
5.8 \times 10^{-4} \mathrm{~s}^{-1} \\
6.9 \times 10^{-2} \mathrm{~s}^{-1} \\
1.4 \times 10^{-1} \mathrm{~s}^{-1}\end{array}$ & $\begin{array}{l}\text { [Siemers et al., 1997] }]^{b} \\
\text { [Siemers et al., 1997] } \\
{[\text { Siemers et al., 1997] }]^{\mathrm{b}}} \\
\text { [Svensson et al., 1995] }]^{\mathrm{d}} \\
\text { [Robert et al, 1982] }]^{\mathrm{d}}\end{array}$ \\
\hline $\begin{array}{l}D_{C} \\
D_{P} \\
D_{D}\end{array}$ & $\begin{array}{l}8.0 \times 10^{-6} \mathrm{~mm}^{2} \mathrm{~s}^{-1} \\
9.4 \times 10^{-4} \mathrm{~mm}^{2} \mathrm{~s}^{-1} \\
2.0 \times 10^{-3} \mathrm{~mm}^{2} \mathrm{~s}^{-1}\end{array}$ & $\begin{array}{l}\text { [Nugent and Jain, } 1984]^{c} \\
\text { [Nugent and Jain, } 1984]^{c} \\
\text { [Nugent and Jain, 1984] }]^{c}\end{array}$ \\
\hline $\begin{array}{l}k_{a} \\
k_{d}\end{array}$ & $\begin{array}{l}3.88 \times 10^{5} \mathrm{M} \mathrm{s}^{-1} \\
4.83 \times 10^{-4} \mathrm{~s}^{-1}\end{array}$ & $\begin{array}{l}\text { [Jackson et al. 1998b] } \\
\text { [Jackson et al. 1998b] }\end{array}$ \\
\hline $\begin{array}{l}P_{C} \\
P_{P} \\
P_{D}\end{array}$ & $\begin{array}{l}9.0 \times 10^{-6} \mathrm{~mm} \mathrm{~s}^{-1} \\
2.2 \times 10^{-3} \mathrm{~mm} \mathrm{~s}^{-1} \\
4.7 \times 10^{-3} \mathrm{~mm} \mathrm{~s}^{-1}\end{array}$ & $\begin{array}{l}\text { [Baxter and Jain, } 1996] \\
\text { [Jain, 1987] } \\
\text { [Jain, 1987] }\end{array}$ \\
\hline $\begin{array}{l}A_{0} \\
V_{\max } \\
K_{m i} \\
a_{I} \\
b_{I}\end{array}$ & $\begin{array}{l}1.0 \times 10^{-8} \mathrm{M} \\
232 \mathrm{~s}^{-1} \\
1.9 \times 10^{-5} \mathrm{M} \\
2.5 \times 10^{-4} \mathrm{~s}^{-1} \\
0.2 .5 \times 10^{-5} \mathrm{~s}^{-1}\end{array}$ & 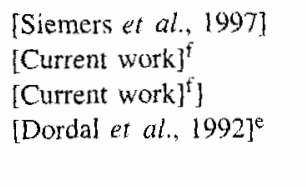 \\
\hline
\end{tabular}

Experimental data show that the intracellular concentration of doxorubicin reaches a steady state
Based on an injection of $1 \mathrm{mg} / \mathrm{kg}$ conjugate $\left(M_{*}=63 \mathrm{kD}\right.$ ) and a blood volume of $2.5 \mathrm{ml}$;

${ }^{6} \mathrm{~A}$ least-squares fit for the blood clearance of $\mathrm{L}-49-\mathrm{sFV}$-bL, was used to compute the compartmental transfer coefficients and the blood elimination rate, $k_{12}, k_{21}$, and $k_{\mathrm{e}}$ [Siemers ef al, 1997];

Calculated as $a\left(M_{W}\right)^{b}$ with values for $a$ and $b$ taken from [Nugent and Jain, 1984\};

"Calculated from the $\alpha$-half life reported in [Svensson $e$ t $a l ., 1995$ ] and [Robert et al., 1982];

eThis parameter was estimated using value for cellular permeability published in [Dordal et al., 1902]:

tThese yalues were obtained from experiments performed concurrently for the purpose of testing the mathematical model.

after 1-2 hours [Paul et al., 1979], [DeGregorio et al., 1984]. The parameter, $b_{I}$, representing a small amount of breakdown or escape from the cell is included in the model for completeness.

\section{Theoretical Analysis}

The system was non-dimensionalized by timescale of conjugate association $(\approx O(\min ))$ in order to be consistent with [Jackson et al., 1999a]. On the timescale of interest, diffusion of conjugate is a relatively slow process $(\approx O(h))$ making the quasi-steady limit used by many authors to analyze reaction diffusion 
processes inappropriate for this system. The transfer of molecules from the tumor back into the plasma is also a slow process $(\approx O(h))$ and therefore these terms can be neglected to first order. The nondimensionalization and the resulting equations can be found in Appendix I. Note that the equations governing the first step of treatment, conjugate localization, are decoupled from the equations describing the prodrug administration and subsequent drug production. In [Jackson et al., 1999a], we studied this decoupled system in detail using singular perturbation techniques to derive a uniformly valid solution. Slow diffusion on the timescale of interest results in a small parameter $\epsilon=\left(D_{C} / k_{a} A_{0} L^{2}\right) \approx 0.1$. To first oder, with the reaction in equilibrium, it was determined that the concentration of free conjugate in tumor can be approximated by

$$
\begin{aligned}
u(r, t) \approx & \frac{A \mu}{\xi_{1}-\mu}\left[e^{-\mu t}-e^{-\xi_{1} t}\right] \\
& +\frac{\mu B}{\xi_{2}-\mu}\left[e^{-\mu t}-e^{-\xi_{2} t}\right]
\end{aligned}
$$

where $w_{1}(t)=A e^{-\xi_{1} t}+B e^{-\xi_{2} t}$ representing a bolus injection into the blood, $\mu=(\bar{\nu} /(1+1 / \zeta))$, and $\bar{\nu}=$ $\left(P / L k_{0} A_{0}\right)$.

To analyze the prodrug and drug dynamics in the blood the following simplifying assumptions were made.

- Clearance is so rapid that levels are not measurable in the normal tissues leaving only blood elimination and production/conversion in the equations. Experiments conducted for this study show that levels of prodrug (CDox) and drug (Dox) in the blood fall below measurable quantities as early as two hours post-injection [Paul et al, 1979], [DeGregorio et al., 1984].

- The ratio of the initial injected concentration of prodrug and the enzyme kinetic parameter, $\delta$, is chosen to be small enough to be ignored.

The equations become

$$
\begin{aligned}
& \frac{d y_{1}}{d t}=-\bar{\kappa} y_{1}-\lambda w_{1} y_{1} \\
& \frac{d z_{1}}{d t}=-\tilde{\kappa} z_{1}+\lambda w_{1} y_{1}
\end{aligned}
$$

and the solutions with $\delta$ negligible are

$$
\begin{aligned}
& y_{1}(t)=e^{-\bar{\kappa} t-\lambda \int^{t} w_{1}(r) d r} \\
& z_{1}(t)=c e^{-\bar{\kappa} t}+e^{-\tilde{\kappa} t} \int^{t} \lambda w_{1}(s) y_{1}(s) e^{\tilde{\kappa} s} d s
\end{aligned}
$$

where the constant, $c$, is found by imposing the initial condition. A strictly asymptotic expansion of the integral $I(t)=\int^{t} w_{1}(s) y_{1}(s) e^{\tilde{\kappa} s} d s$ can be derived by repeated integration by parts.

$$
\begin{aligned}
I(t) & =\frac{y_{1}(t) w_{1}(t) e^{\tilde{\kappa} t}}{\tilde{\kappa}-\bar{\kappa}-\xi_{2}}+\ldots \\
& =\frac{\lambda B}{\tilde{\kappa}-\vec{\kappa}-\xi_{2}} y_{1}(t) e^{-\left(\xi_{2}-\tilde{\kappa}\right) t}
\end{aligned}
$$

where the plasma dynamics are assumed to behave as $\omega_{1} \approx B e^{-\xi_{2} t}$ which represent the long time behavior of conjugate elimination from the blood. We do this because in $\omega_{1}=A e^{-\xi_{1} t}+B e^{-\xi_{2} t}, \xi_{1} \gg \xi_{2}$, and prodrug administration occurs hours or days after conjugate administration making the first exponential negligible. If the first term of the asymptotic expansion is used to approximate the integral, the solutions become

$$
\begin{aligned}
& y_{1}(t)=e^{-\bar{\kappa} t-\lambda \int^{t} \omega(r) d r} \\
& z_{1}(t)=\frac{\lambda}{\tilde{\kappa}-\tilde{\kappa}-\xi_{2}}\left(y_{1}(t) \omega_{1}(t)-B e^{\tilde{\kappa} t}\right)
\end{aligned}
$$

In the tumor, the same assumption relating the initial injected dose of prodrug, $P_{0}$ to the enzyme kinetic parameter $K_{M}$ is imposed as well as the assumption that $\left(b_{I} / K_{M}\right) \ll 1$ resulting in

$$
\begin{aligned}
\frac{\partial p}{\partial t}= & \sigma_{1} \Delta p-\lambda(u(r, t)+v(r, t)) p \\
\frac{\partial d_{E}}{\partial t}= & \sigma_{2} \Delta d_{E}+\lambda(u(r, t) \\
& +v(r, t)) p-\alpha d_{E} \\
\frac{\partial d_{I}}{\partial t}= & \alpha d_{E} \\
p(r, 0)= & 0 \quad d_{E}(r, 0)=0 \quad d_{I}(r, 0)=0 \\
\frac{\partial p}{\partial r}(1, t)= & 0 \quad \frac{\partial d_{E}}{\partial r}(1, t)=0
\end{aligned}
$$


100

$$
\begin{gathered}
\frac{\partial p}{\partial r}(\bar{\rho}, t)=-\nu_{2}\left(y_{1}(t)-p(\bar{\rho}, t)\right) \\
\frac{\partial d_{F}}{\partial r^{r}}(\bar{\rho}, t)=-\nu_{3}\left(z_{1}(t)-d_{E}(\bar{\rho}, t)\right)
\end{gathered}
$$

These equations for the concentrations of prodrug and drug in the tumor decouple from the blood dynamics except at the capillary tissue interface. Also, the equation for the prodrug concentration (the first of (16)) is independent of the concentration of drug in the tumor (the second of (16)). Analytical solutions for the prodrug concentration in the tumor can be derived for certain special cases; unfortunately, such analytical solutions are of little use (except asymptotically) in obtaining numerical values for the intratumoral prodrug and drug concentrations.

\section{RESULTS}

\section{Conjugate in Tumor}

Figure 2 shows a comparison of the analytical solution of conjugate concentration in the tumor, (12), to the numerical solution of the original, dimensional system of equations $1,4,7,8$. The comparison is made by calculating the average concentration over the volume of the cylinder and using this result to compute the percent injected dose of conjugate per gram of tumor tissue. Figure 2 also shows that the assumption of reaction equilibrium is valid for long times. The fact that the ordinary differential equation approximate solution was used results in an analytical expression that consistently underestimates the numerical solution. However, the time of

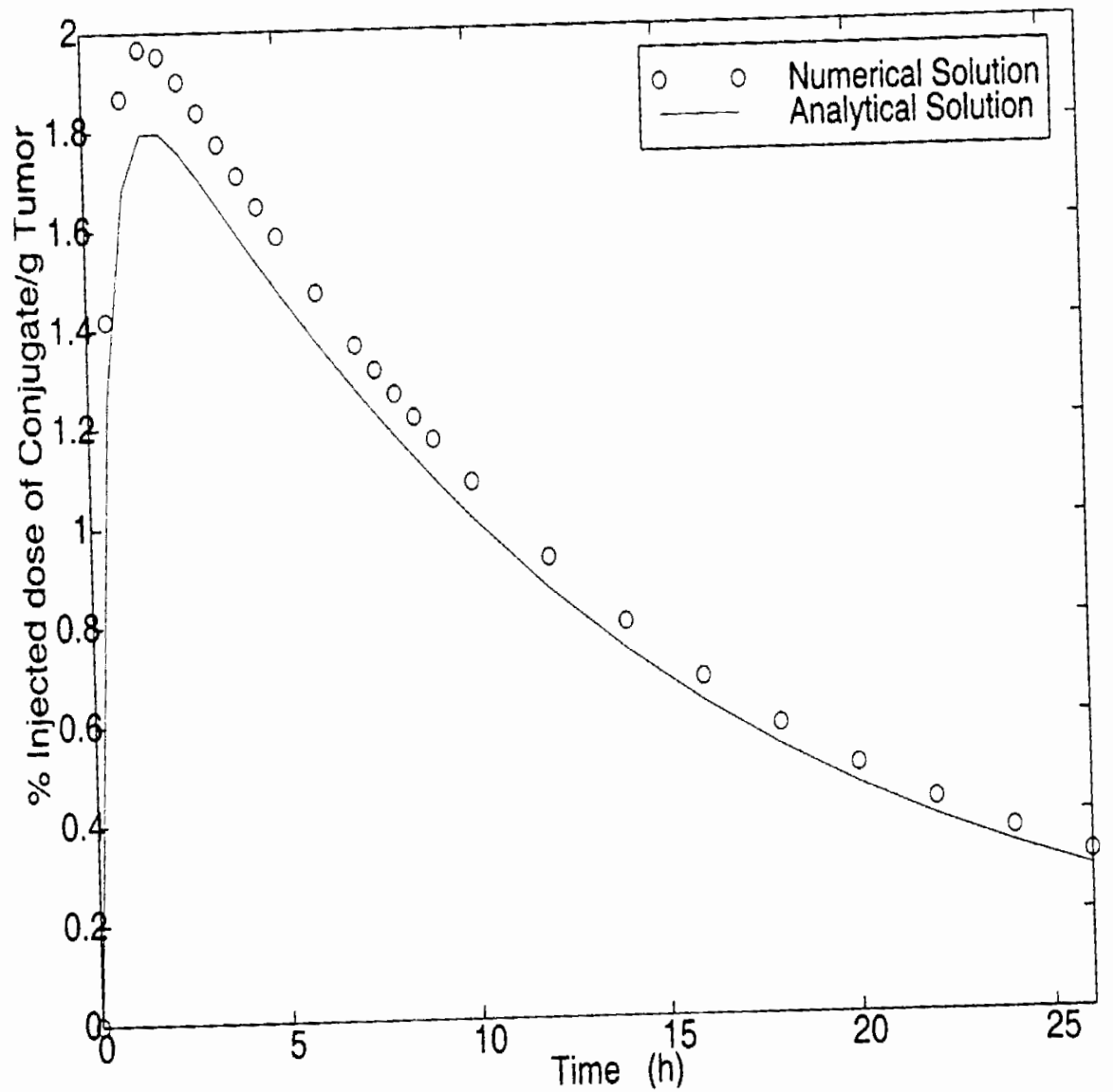

FIGURE 2 Comparison of numerical simulations for conjugate levels in the tumor (equations $1,4,7,8$ ) with the analytic approximation derived in equation 20 . 
the peak was captured quite well. The result in (12) allows certain clinically important quantities to be approximated analytically [Jackson et al., 1999a].

\section{Prodrug and Drug in the Blood}

Figure 3 shows how well the analytical approximations for prodrug levels in the blood given by (15) compare with the numerical solution of (13) when the nonlinearity is included. The value of $\delta$ in the simulations was 0.3 and because (15) is an asymptotic approximation, the analytical result in Figure (3b) is an underestimation of numerical solution for short times. Equation (14), demonstrates that the concentration of prodrug in the blood is influenced most by the initial injected

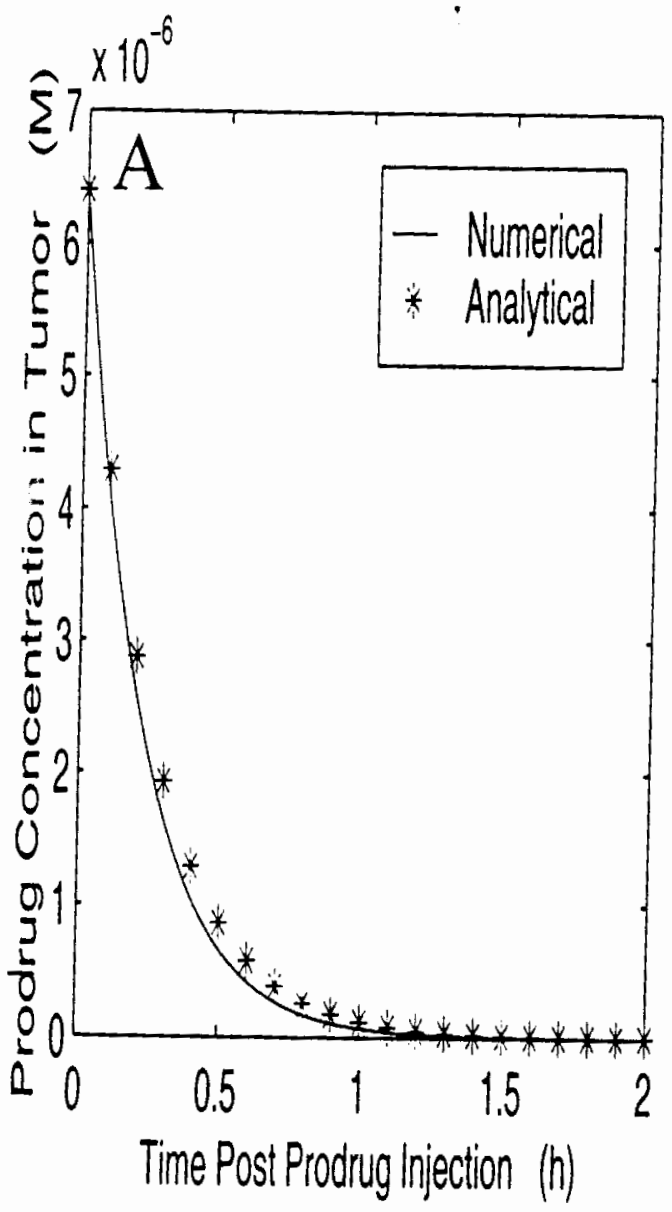

dose, the clearance rate, and the concentration of conjugate in the blood. Drug concentrations, on the other hand, are affected most by changes in prodrug and drug clearance rates, the parameter $\lambda=\left(V_{\max } F_{0} / k_{a} A_{0} K_{M}\right)$, and the conjugate and prodrug concentrations in the blood.

\section{Dependence on Tumor Vasculature and Time of Administration}

As with the conjugate, the prodrug concentrations in the tumor decrease with inter-capillary half distance and this dependence is less significant over time (Figure 4). Drug levels, on the other hand, are higher in poorly vascularized tissue and reflect more strongly the conjugate distribution. Again,

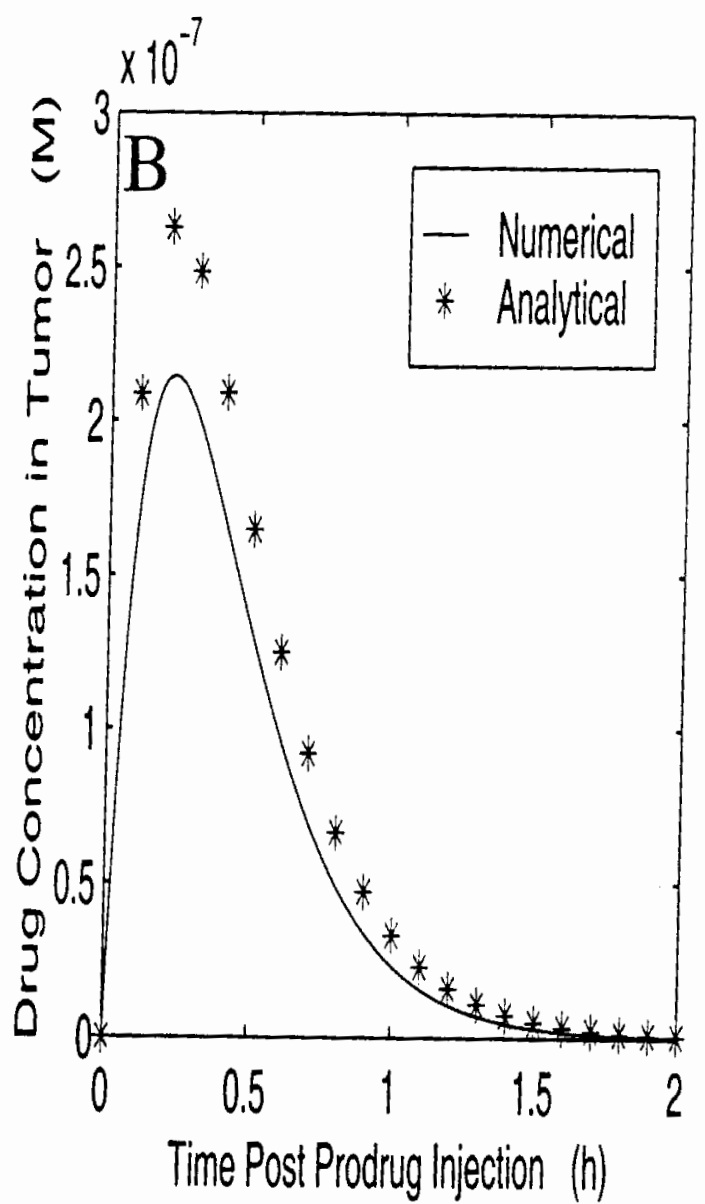

FIGURE 3 Comparison of the numerical solution of equation (13) governing prodrug and drug concentrations in the blood with the analytical approximations, equation (15). In these simulations $\delta=0.3$. 


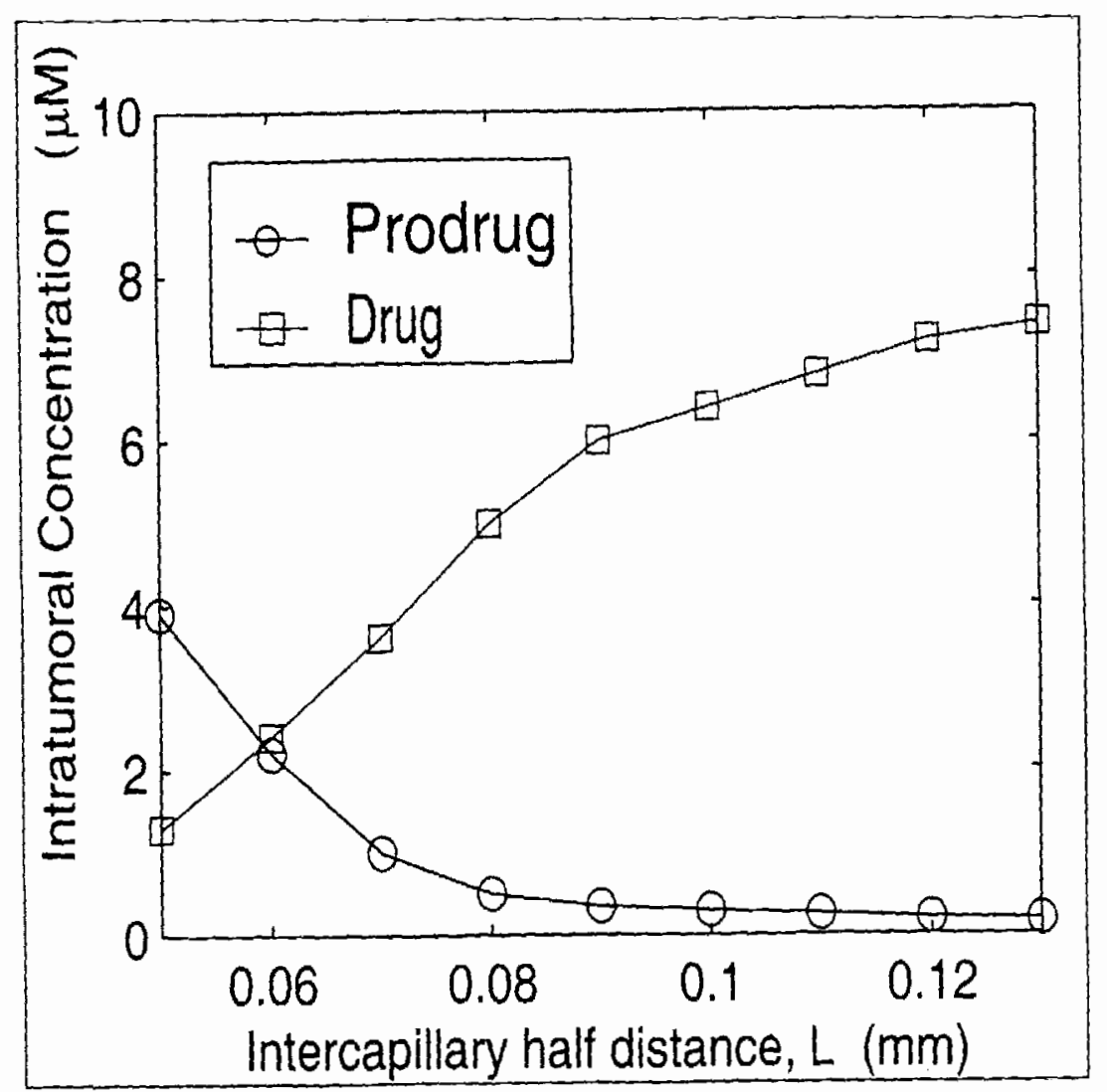

FIGURE 4 Dependence of prodrug and drug concentrations in the tumor on the inter-capillary half distance, $L$. The levels were measured one hour post prodrug administration. The parameters used in this simulation are given in Table III.

this dependence on the inter-capillary half distance diminishes over time. Figure 4 shows the dependence of both prodrug and drug concentrations in the tumor on the inter-capillary half distance when level are measured one hour post injection.

The time of prodrug administration also affects the drug concentration in both the blood and the tumor. The drug concentration in the blood and in the tumor at various times after prodrug administration are shown in Figure 5. With a short time delay between conjugate and prodrug administration, blood levels of conjugate are higher leading to much higher drug concentrations in the blood (Figure 5A). The longer the delay, the lower the drug concentration in the blood. In the tumor, however, the dependence on the time of prodrug administration is less significant (Figure 5B). The levels of drug are only moderately higher when the delay between conjugate and prodrug administration is short.

\section{Critical Parameters for Increased Intratumoral Drug Production}

The effect of each parameter $(E)$ on the area under the curve $(A U C)$ of intratumoral drug concentrations over time was investigated by calculating $E_{A U C}=\left(\left(A U C_{2}-A U C_{1 / 2}\right) / A U C\right)$. Here $A U C$ is the area under the curve when all parameters have their baseline values listed in Table III, $A U C_{2}$ and $A U C_{1 / 2}$ are the areas under the curve associated with reducing by half and doubling each parameter respectively. The baseline $A U C$ when parameters have the values listed in Tabie III was $3.3 \times 10^{-5} \mathrm{Mh}^{-1}$. The results of these simulations are listed in Table IV. 

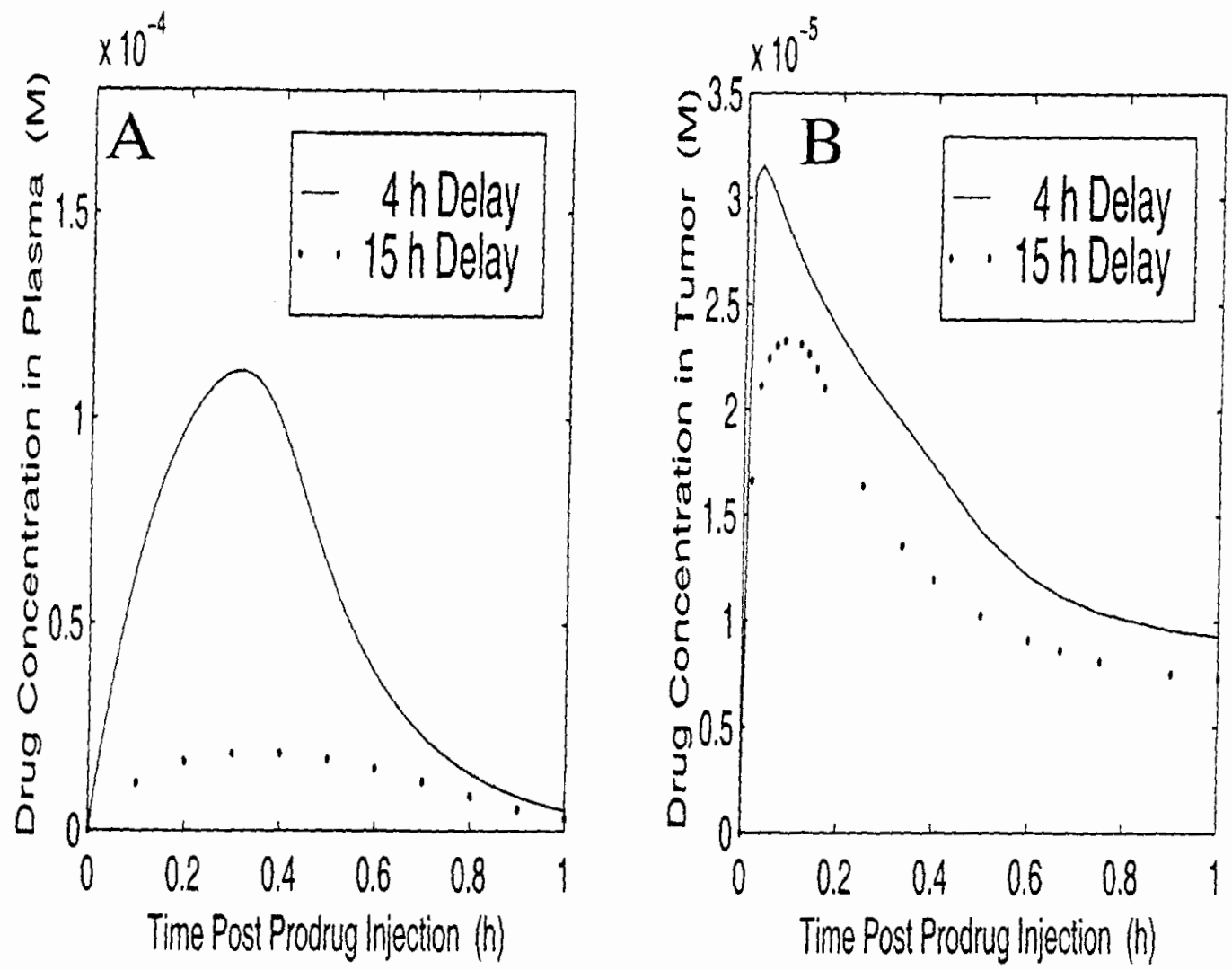

FIGURE 5 Plot of prodrug and drug concentrations in the tumor vs. time while varying the time delay between conjugate and prodrug administration. The dependence on this delay is more pronounced for drug level in the blood and less significant intratumoral drug production. The parameter used in this simulation are given in Table III.

T. : most significant pharmacokinetic parameter for intratumoral drug production was the rate of prodrug clearance from the blood, $\bar{k}_{\varepsilon}$. Reducing prodrug blood clearance allows more prodrug to enter the tumor mass and results in higher drug concentrations over time. The dominant clinical parameter was the initial injected dose of prodrug, $P_{0}$. As expected, higher initial doses leads to higher intratumoral drug concentrations. Increasing the initial injected dose of conjugate has the same effect, however less pronounced. The enzyme kinetic parameters also play a role, in that increasing the prodrug conversion rate, $V_{\max }$ results in higher drug concentrations over time.

Of all the physiological parameters tested, the prodrug $\left(P_{P}\right)$ and drug $\left(P_{D}\right)$ permeability had the most pronounced effect on the $A U C$ in the tumor. Increasing the prodrug permeability and therefore allowing more to penetrate the capillary and enter the tumor leads to higher drug levels in the tumor. In contrast, decreasing the drug permeability and thus trapping the agent in the tumor results in significantly higher values for the intratumoral drug $A U C$.

It is particularly important to consider the targeting index $\left(T I=\left(A U C_{\text {tumor }} / A U C_{\text {blood }}\right)\right)$ which measures the overall exposure to drug and how well the drug is targeting the tumor. The effect of each parameter on the (TI) was investigated and the results are presented in Table $V$. Of all the parameters tested, the rate at which the conjugate is eliminated from the blood $\left(k_{e}\right)$ had the most influence on the targeting index. Clearing the conjugate from the blood dramatically increases $T I$. Increasing the drug elimination rate in the blood $\left(\tilde{k}_{e}\right)$ will have similar effects, however less pronounced. Decreasing the initial injected dose of conjugate, $C_{0}$, and the enzyme conversion parameter $V_{\max }$ can 
TABLE IV The effect of each parameter on the area under the average drug concentration vs. time curve, $A U C$, in the tumor

\begin{tabular}{|c|c|c|c|c|}
\hline & & $A U C_{1 / 2}\left(10^{-5} \mathrm{Mh}\right)^{\mathrm{a}}$ & $A U C_{2}\left(10^{-5} \mathrm{Mh}\right)^{\mathrm{a}}$ & $E^{b}$ \\
\hline \multicolumn{5}{|c|}{ Phamacokinetic } \\
\hline \multicolumn{5}{|c|}{ Parameters } \\
\hline & $\bar{\kappa}\left(\mathrm{h}^{-1}\right)$ & 4.5 & 2.2 & -0.70 \\
\hline & $\bar{\kappa}\left(\mathrm{h}^{-1}\right)$ & 3.4 & 3.2 & -0.06 \\
\hline & $k_{12}\left(\mathrm{~h}^{-1}\right)$ & 3.4 & 3.2 & -0.06 \\
\hline & $k_{21}\left(\mathrm{~h}^{-1}\right)$ & 3.2 & 3.5 & 0.07 \\
\hline & $k_{e}\left(\mathrm{~h}^{-1}\right)$ & 3.6 & 2.4 & -0.4 \\
\hline & $k_{a}\left(\mathrm{M}^{-1} \mathrm{~h}^{-1}\right)$ & 3.2 & 3.3 & 0.03 \\
\hline & $k_{d}\left(\mathrm{~h}^{-1}\right)$ & 3.3 & 3.2 & -0.03 \\
\hline \multicolumn{5}{|l|}{ Clinical } \\
\hline \multicolumn{5}{|l|}{ Parameters } \\
\hline & $C_{0}(\mathrm{M})$ & 2.3 & 3.7 & 0.4 \\
\hline & $P_{0}(\mathrm{M})$ & 1.8 & 4.7 & 0.9 \\
\hline & $A_{0}(\mathrm{M})$ & 3.2 & 3.3 & 0.03 \\
\hline \multicolumn{5}{|l|}{ Enzyme } \\
\hline \multicolumn{5}{|l|}{ Properties } \\
\hline & $V_{\max }\left(\mathrm{h}^{\mathrm{l}}\right)$ & 2.2 & 3.8 & 0.5 \\
\hline & $K_{N}(\mathrm{M})$ & 3.5 & 3.0 & -0.2 \\
\hline \multicolumn{5}{|l|}{ Physiological } \\
\hline \multicolumn{5}{|l|}{ Parameters } \\
\hline & $D_{C}\left(\mathrm{~mm}^{2} \mathrm{~h}^{-1}\right)$ & 3.2 & 3.3 & 0.03 \\
\hline & $D_{p}\left(\mathrm{~mm}^{2} \mathrm{~h}^{-1}\right)$ & 3.2 & 3.3 & 0.03 \\
\hline & $D_{D}\left(\mathrm{~mm}^{2} \mathrm{~h}^{-1}\right)$ & 3.0 & 3.4 & 0.1 \\
\hline & $P_{C}\left(\mathrm{~mm} \mathrm{~h}^{-1}\right)$ & 3.4 & 2.7 & -0.2 \\
\hline & $P_{P}\left(\mathrm{~mm} \mathrm{~h}^{-1}\right)$ & 1.9 & 4.3 & 0.7 \\
\hline & $P_{D}\left(\mathrm{~mm} \mathrm{~h}^{-1}\right)$ & 5.7 & 2.0 & -1 \\
\hline & $a_{r}\left(\mathrm{~h}^{-1}\right)$ & 2.8 & 4.2 & 0.4 \\
\hline & $b_{Y}\left(\mathrm{~h}^{-1}\right)$ & 3.3 & 3,3 & 0 \\
\hline
\end{tabular}

${ }^{a} A U C_{1 / 2}$ and $A U C_{2}$ are the areas under the average drug concentration vs. time curve associated with teducing by half and doubling. respectively, the specific parameter listed;

${ }^{b} T 0$ measure the effect of each parameter on the area under the average drug concentration vs. time curve in the tumor, the ratio $E=\left(\left(A U C_{2}-A U C_{12}\right) / A U C_{1}\right)$ was computed, where $A U C_{1}=3.3 \times 10^{-5} \mathrm{M} \mathrm{h}^{-1}$ is the area under the curve when all parameters have their baseline values. The larger the deviation from zero, the more significant the parameter

greatly increase $T I$ due to the reduced levels of drug produced in the blood.

\section{Correlation Between the Mathematical Model and the Experimental Data}

In order to validate the mathematical model, in vivo studies were undertaken to determine the intratumoral and blood quantities of prodrug and drug in mice that had subcutaneous 3677 human melanoma tumor xenografts. At a point when the tumors were approximately $250 \mathrm{~mm}^{3}$ in size, the animals were treated with a single bolus injection of $4 \mathrm{mg} / \mathrm{kg}$ of L49-sFv-bL, a recombinant fusion protein [Siemers et al., 1997] that binds to cell surface antigens on the melanoma tumor implants. After waiting $15 \mathrm{~h}$ for tumor localization and blood clearance to occur, the prodrug CDox was administered at a dose of $80 \mathrm{mg} / \mathrm{kg}$. At various time points, the tumors and blood were removed and extracted. The amount of Dox and CDox was measured by high pressure liquid chromatography according to a previously published procedure [Svensson et al., 1995] The data obtained were compared to the mathematical model simulations and an excellent correlation was found to exist for prodrug levels in the tumor and in the plasma; as well as for drug levels in the plasma (Figure 6A, 6B, 6C). The model also 

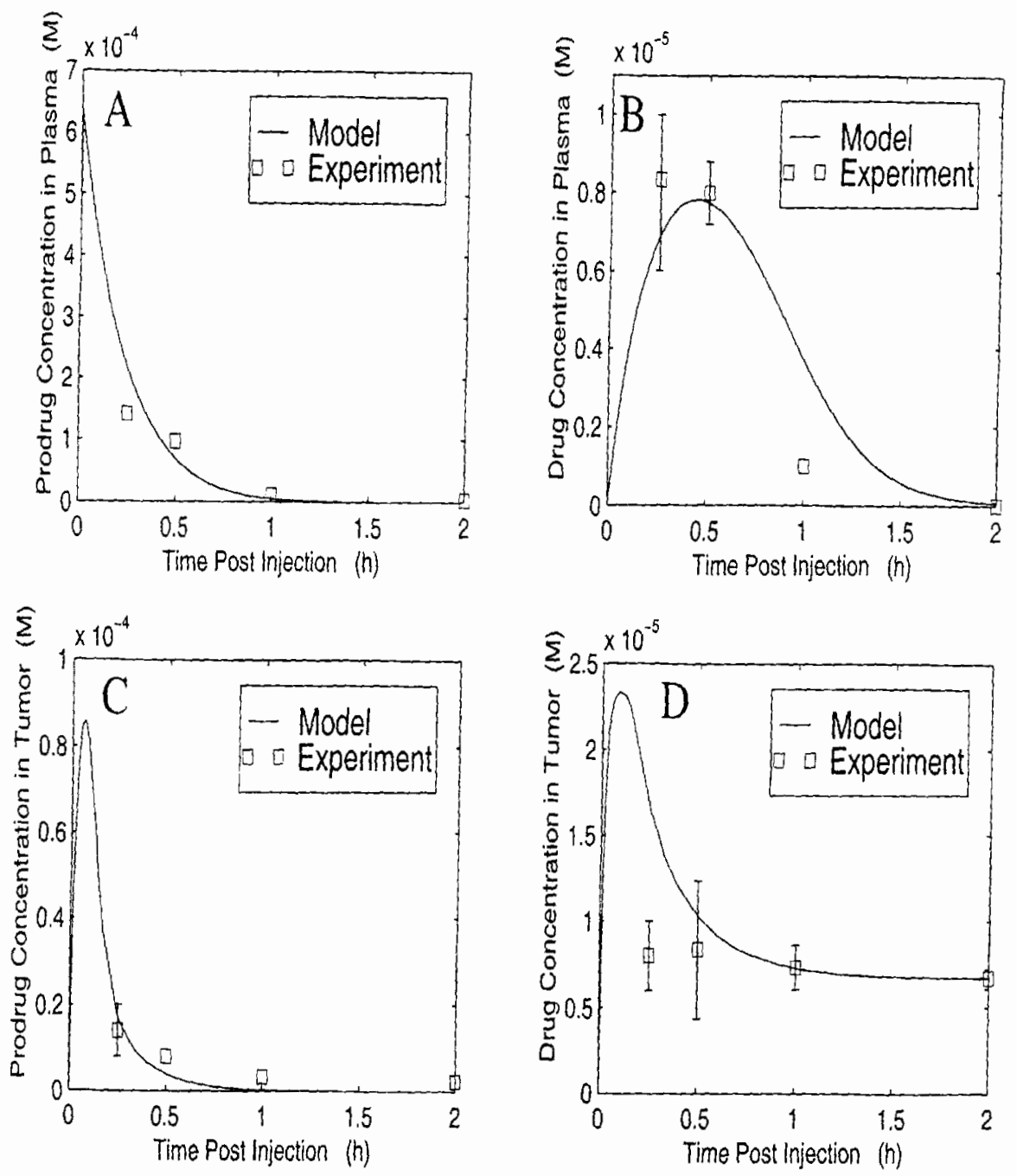

FIGURE 6 Comparison of numerical solution of the mathematical model equations $(2,3,5,6,9-11)$ which describe the prodrug and drug concentrations in both the blood and in the tumor with experimental data. Mice with 3677 melanoma rumors were treated with L49-SFv-bL and then with the prodrug CDox. The amount of CDox in (A) the plasma, (C) the tumor, and the amount of active drug Dox in (B) the plasma, and (D) the tumor was quantified experimentally.

captures the long time experimental profiles for drug concentration in the tumor (Figure 6D). The discrepancy in the short time behavior might be the result of several factors including the fact that the rate drug generation may be different in the tissue.

\section{TUMOR HETEROGENEITY}

Until now, the tumor has been assumed to be a homogeneous collection of Krogh tissue cylinders with average distance between capillary centers of $L=0.2 \mathrm{~mm}$. This average does not account for the fact that the intercapillary half distance can vary widely within a solid tumor mass. In addition to the structural heterogeneity, a series of papers by Baxter and Jain presented mathematical models that showed how the absence of lymphatics in tumors, combined with high tumor vascular hydraulic conductivity, leads to high interstitial pressure in the tumor interior, decreasing to the level of the surrounding tissue in a thin boundary layer at the tumor periphery 
[Baxter and Jain, 1989]. This high interior pressure has two effects: (1) a greatly reduced pressure difference for interior vessels, leading to very low convective transport (i.e. diffusive flux is the only mechanism for transport of macromolecules across vessel walls), (2) a convective current directed out of the tumor. This suggests that convection acts to impede the diffusion of macromolecules into the tumor.

The interstitial pressure boundary layer is estimated to be about $0.03 \mathrm{~cm}$ [Baxter and Jain, 1989] which correlates to the well vascularized layer on the outer edge of the tumor that is estimated to be 0.01-0.02 cm thick in [Endrich et al., 1979 (See Figure 6). In this section the previous mathematical model in $(1-11)$ is adapted to include these features inherent in tumor's structure and composition by adding a term for the convective flux of conjugate, prodrug, and drug directed out of the tumor. The resulting model equations can be found in Appendix II. An expression for the interstitial convective velocity within an isolated tumor, $c$, was derived in [Baxter and Jain, 1989]. The authors made use of Darcy's law for flow through a porous medium and a continuity equation for steady-state incompressible flow to obtain an equation for the interstitial pressure. From this they determined the interstitial velocity to behave as

$$
\begin{aligned}
c(r)= & \frac{K\left(p_{e}-p_{\infty}\right)}{r^{2}} \\
& \times\left[\frac{\alpha \cosh \alpha r / R-R \sinh \alpha r / R}{\sinh \alpha}\right]
\end{aligned}
$$

where $K$ is the hydraulic conductivity of the interstitium $\left(\mathrm{cm}^{2} / \mathrm{mm} \mathrm{Hg}-\mathrm{sec}\right), p_{e}$ is the effective vascular pressure $(\mathrm{mm} \mathrm{Hg}), p_{\infty}$ is the interstitial fluid pressure in the surrounding normal tissue ( $\mathrm{mm} \mathrm{Hg}$ ), and $\alpha$ is a dimensionless ratio relating interstitial to vascular fluid flow.

We consider a spherical tumor consisting of a heterogeneous collection of Krogh tissue cylinders, The radius of the tumor is taken to be $R=3.5 \mathrm{~mm}$ giving a total volume of $180 \mathrm{~mm}^{3}$ which correlates to the size of the tumors at the initiation of treatment in [Kerr et al, 1995]. The tumor is divided into three regions, a well vascularized periphery, an intermediate region of average vascularity, and a semi-necrotic center. The convective velocity will differ in each region of the tumor and we use equation (18) to determine estimates for the velocity at different radial positions. The cross-section of the model tumor is shown in Figure 6, where $r^{\prime}$ is the large scale spatial variable measured from

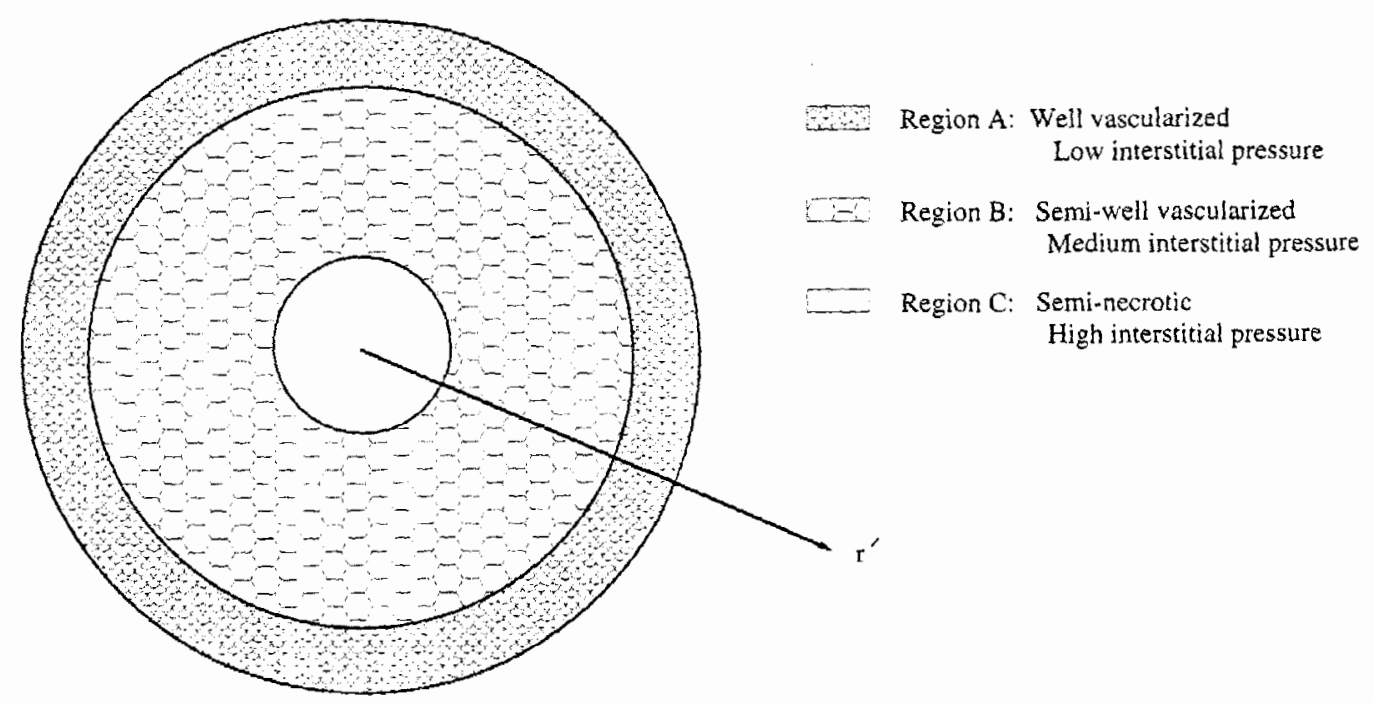

FIGURE 7 Cross-section of spherical, heterogeneous tumor to be used in numerical simulations. Region $A$ on the periphery of the tumor contains low pressure and abundant vascularization; where as regions $B$ and $C$ in the tumor interior have increased interstitial pressure and poorer vascularity. 
the center of the sphere. The local, small scale spatial variable for each Krogh cylinder radius will remain, $r$. We consider independent Krogh cylinder models in each region of the tumor with no coupling between regions. Krogh cylinders taken from the well vascularized periphery of the tumor (Region A, $r^{\prime}=2.9-3.5 \mathrm{~mm}$ ) have small intercapillary half distances, $L$, and negligible outward convection, $c$. Tissue cylinders removed from the intermediate section of average vascularity and interstitial pressure (Region B, $r^{\prime}=0.9-2.9 \mathrm{~mm}$ ) have average $L$ and $c$ values and cylinders from the semi-necrotic region with few blood vessels in addition to the high interstitial pressure (Region $\mathrm{C}$, $r^{\prime}=0-0.9 \mathrm{~mm}$ ) have large distances between capillaries and larger convection velocities.

\section{Numerical Simulations}

The effects of tumor heterogeneity and increased interstitial pressure on the localization and retention of conjugate in the tumor were initially investigated. Table VI shows the parameter values used in the

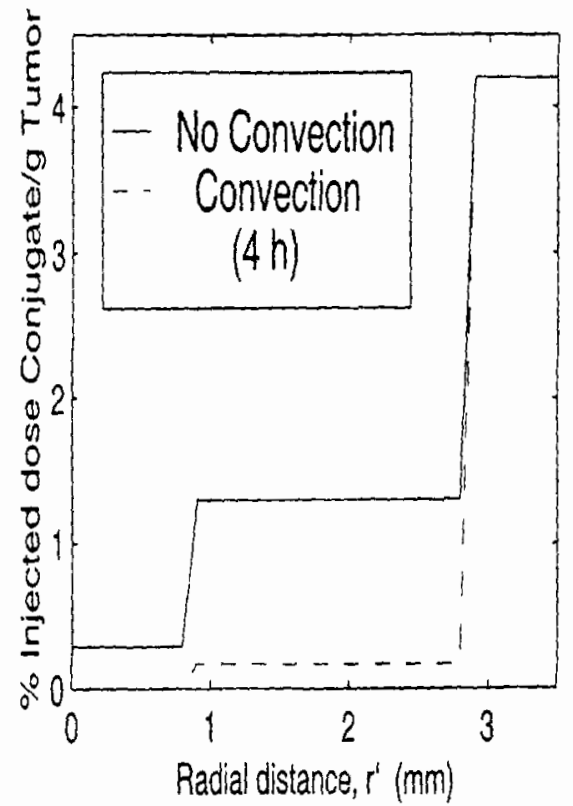

numerical simulation for each region of the tumor. Parameters that are not mentioned were given their baseline values listed in Table III. Recall, that regions of poor vascularity tend also to be regions of increased interstitial pressure. The fact that the pressure in the tissue is higher than that in the capillary leads to a convective current directed out of the tumor. This suggests that convection acts to impede the diffusion of macromolecules into the tumor. Figure 8 shows the effects of vascular heterogeneity and outward convection on intratumoral conjugate concentrations. Numerical simulations show that increased interstitial pressure and convection can lead to 70-90\% less conjugate in the interior of the tumor than observed when convection is ignored. This prediction of the model is readily testable for certain fluorescent drugs such as doxorubicin as its location in the tumor can easily be detected.

It is clear that vascular heterogeneity and increased interstitial pressure can significantly affect the concentration of conjugate in the tumor. The effect of these properties on drug generation and retention is also noteworthy. Figure 9 shows the drug concentration profiles in a cross section of the

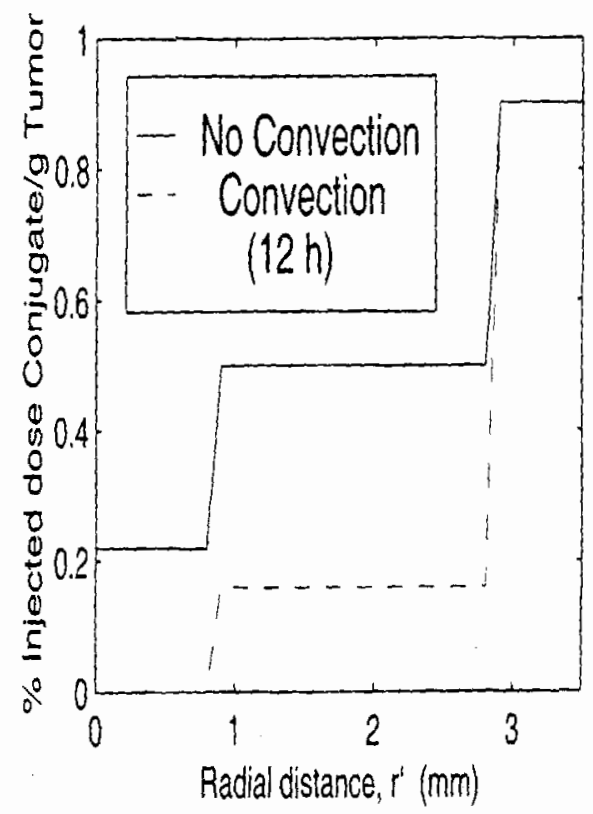

FIGURE 8 Conjugate concentration profiles through a cross-section of a spherical tumor when both vascular heterogeneity and outward convection are considered. Region $\mathrm{A}$ is well vascularized with no outward convection, region $\mathrm{B}$ is semi-well with small outward convection vascularized, and region $\mathrm{C}$ is semi-necrotic with large outward convection. 

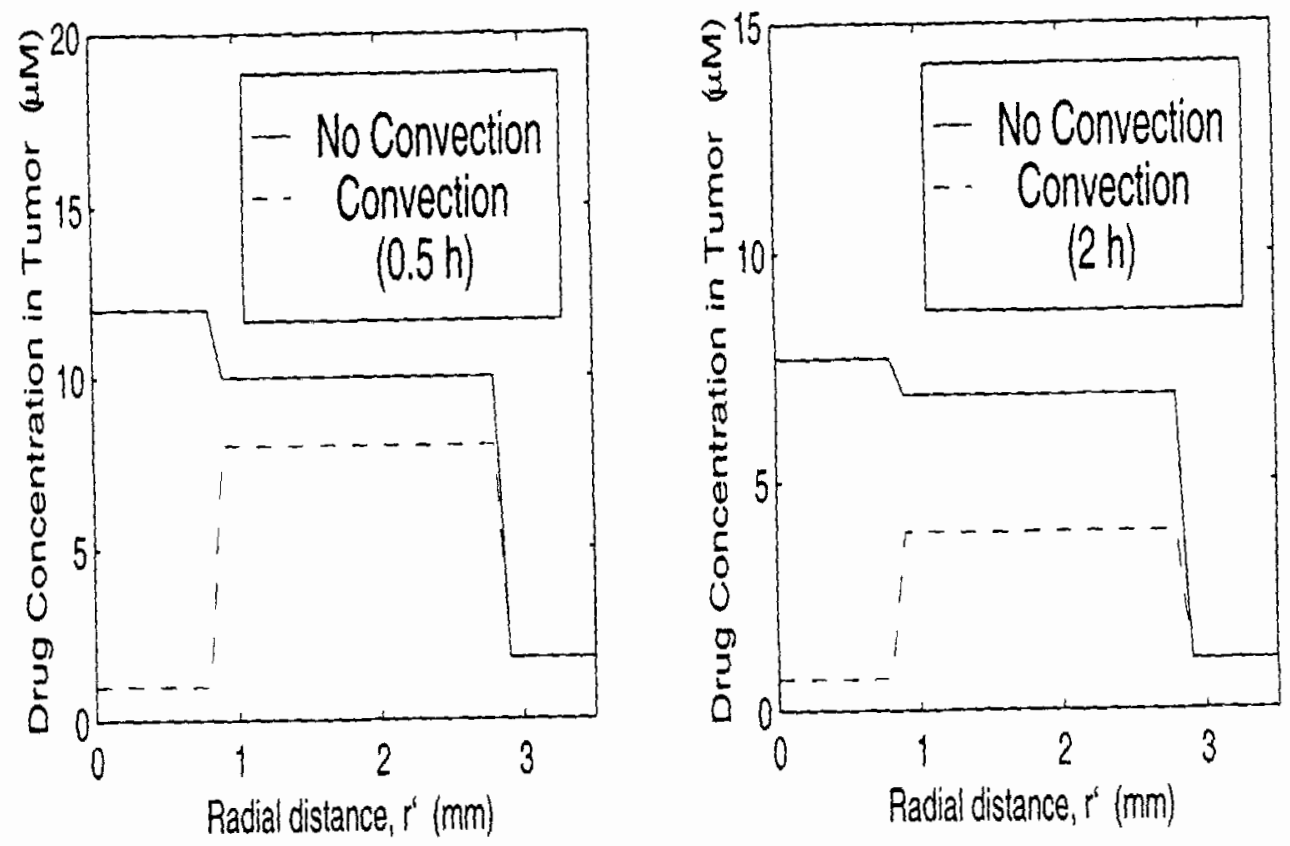

FIGURE 9 Drug concentration profiles through a cross-section of a spherical tumor when both vascular heterogeneity and outward convection are accounted for, Region $A$ is well vascularized with no outward convection, region $B$ is semi-well with small outward convection vascularized, and region $\mathrm{C}$ is semi-necrotic with large outward convection.

tumor when vascular heterogeneity and the effects of increased interstitial pressure are included. The numerical simulations predict that it is not the well vascularized or the semi-necrotic regions, but the section of average vascularity that will receive the greatest exposure to the drug. Experiments have not been performed on this two-step treatment to determine which section of the tumor receive the most arug, therefore we suggest this as a future direction of experimental investigation.

\section{DISCUSSION}

The use of mAb-enzyme conjugates for selective prodrug conversion has resulted in promising activities both preclinically [Siemers et al., 1997], [Springer et al., 1991], [Meyer et al., 1993, Eccles et al., 1994, Kerr et al., 1995] and clinically [Bagshawe and Begent, 1996]. Conjugate tumor localization and retention is critical for the targeting efficacy in many $\mathrm{mAb}$-based targeting strategies including the two-step strategy described here. A mathematical model that encapsulates the effects that many of the physiological and biochemical parameters have on intratumoral uptake of a mAbenzyme conjugate has previously been reported [Jackson et al,, 1999a]. This continuation study focuses on prodrug treatment after conjugate administration. A mathematical model is developed that describes the conversion of prodrug to active drug in the tumor, blood, and normal tissues, and the intracellulat drug concentrations in the tumor.

Mathematical modeling of prodrug activation produced several significant results. Our model differs in a number of ways from previous studies [Baxter and Jain, 1996, Baxter et al., 1992], including the fact that it accounts for the internalization of drug by the tumor cells. This proved crucial for predicting the experimental results. Simulation of the mathematical model showed that there are several parameters that can significantly influence intratumoral drug production including conjugate and prodrug dose, clearance from the blood, and prodrug and drug permeability. Of these parameters, conjugate and prodrug dose and prodrug and drug 
permeability have the potential to be experimentally controlled. Optimally the prodrug should be designed to be highly permeable leading to increased amounts in the tumor. The released drug should be trapped in the tumor either due to intracellular uptake or to chemical instability.

The targeting index, $T 1$, which measures the ratio of drug production in the tumor to that of the blood can also be significantly improved by varying certain parameters. Conjugate and drug clearance from the blood were most influential in improving this ratio. Decreasing the initial injected dose of conjugate and enzyme conversion parameter, $V_{\max }$, also results in less drug generation in the blood and therefore higher values of $T I$. To counteract a low, $V_{\max }$ a larger dose of prodrug must be administered which also has a positive affect on $T I$. This finding is consistent with studies of [Baxter and Jain, 1996].

Finally, two specific obstacles for the delivery of macromolecules to solid tumors were considered. Vascular heterogeneity and outward convection are investigated together and were found to have a pronounced affect on the conjugate and drug concentrations throughout a nonuniform tumor. For example, there is a $70-90 \%$ decrease in the conjugate levels seen in certain sections of the tumor. The most dramatically different results are in the drug concentration: When increased convection out of the tumor is considered, no longer are the highest levels of drug seen in the poorly vascularized regions of the tumor, but it is the section with average vascularity that maintains the highest levels of conjugate.

One of the most widely tested drug targeting strategies involves the use of direct $\mathrm{mAb}$-drug conjugates [Pietersz et al., 1994]. The distribution of such conjugates within tumors would be expected to resemble that of the mAb-enzyme conjugates described here, since both are macromolecules having similar physical characteristics. In cases where the cytotoxic agent is directly appended to the mAb, the diminishing conjugate concentration deep within the tumor mass undoubtedly will have a pronounced effect on therapeutic efficacy. This concentration gradient may contribute significantly to the lack of clinical efficacy generally observed with direct mAb-drug conjugates used for solid tumor therapy [Pietersz et al., 1994]. The two-step approach described here may circumvent this problem, since the antigen bound mAbenzyme conjugate is used to catalytically generate low molecular weight drugs that can then readily diffuse into the tumor mass. Although the use of $m A b$ enzyme conjugates for prodrug conversion remains largely untested in humans, the mathematical model points to some significant advantages and clinical promise.

\section{APPENDIX I: NONDIMENSIONALIZATION}

The system of equations $(1-11)$ were nondimensionalized with the following rescaling

$$
\begin{aligned}
u & =\frac{C_{F}}{C_{0}} \quad v=\frac{C_{B}}{C_{0}} \quad p=\frac{P}{K_{M}} \\
d_{E} & =\frac{D_{E}}{K_{M}} \quad d_{I}=\frac{D_{I}}{K_{M}} \\
\bar{t} & =k_{a} A_{0} t \quad \bar{r}=\frac{r}{L} \quad \bar{\rho}=\frac{\rho}{L} \\
w_{i} & =\frac{c_{i}}{C_{0}} \quad y_{i}=\frac{p_{i}}{P_{0}} \quad z_{i}=\frac{d_{i}}{P_{0}} \quad i=1,2 \\
\epsilon & =\frac{D_{F}}{k_{a} A_{0} L^{2}} \quad \zeta=\frac{k_{d}}{k_{a} A_{0}} \quad \nu_{1}=\frac{P L}{D_{F}} \quad \gamma=\frac{F_{0}}{b} \\
\sigma_{1} & =\frac{D_{P}}{k_{a} A_{0} L^{2}} \quad \sigma_{2}=\frac{D_{D}}{k_{a} A_{0} L^{2}} \quad \lambda=\frac{V_{\max } C_{0}}{k_{a} A_{0} K_{M}} \\
\delta & =\frac{P_{0}}{K_{M}} \quad \alpha=\frac{a_{I}}{k_{a} A_{0}} \quad \beta=\frac{b_{I}}{k_{a} A_{0}} \\
\nu_{2} & =\frac{P_{F} L}{D_{P}} \quad \nu_{2}=\frac{P_{D} L}{D_{D}}
\end{aligned}
$$

The resulting non-dimensional equations are

$$
\begin{aligned}
& \frac{\partial u}{\partial t}=\epsilon \frac{\partial^{2} u}{\partial r^{2}}+\epsilon \frac{1}{r} \frac{\partial u}{\partial r}-u+\gamma u v+\zeta v \\
& \frac{\partial v}{\partial t}=u-\gamma u v-\zeta v \\
& \frac{\partial p}{\partial t}=\sigma_{1} \frac{\partial^{2} p}{\partial r^{2}}+\frac{\sigma_{1}}{r} \frac{\partial p}{\partial r}-\frac{\lambda(u+v) p}{1+\delta_{p}}
\end{aligned}
$$




$$
\begin{aligned}
& \frac{\partial d_{E}}{\partial t}=\sigma_{2} \frac{\partial^{2} d_{E}}{\partial r^{2}}+\frac{\sigma_{2}}{r} \frac{\partial d_{E}}{\partial r}+\frac{\lambda(u+v) p}{1+\delta_{p}}-\alpha d_{E} \\
& \frac{\partial d_{I}}{\partial t}=\alpha d_{E}-\beta d_{I} \\
& u(r, 0)=0 \quad v(r, 0)=0 \quad p(r, 0)=0 \\
& d_{E}(r, 0)=0 \quad d_{I}(r, 0)=0 \\
& \frac{\partial u}{\partial r}(1, t)=0 \quad \frac{\partial p}{\partial r}(1, t)=0 \frac{\partial d_{E}}{\partial r}(1, t)=0 \\
& \frac{\partial u}{\partial r}(\bar{\rho}, t)=-\nu_{1}[w(t)+u(\bar{\rho}, t)] \\
& \frac{\partial p}{\partial r}(\bar{\rho}, t)=-\nu_{2}[y(t)+p(\bar{\rho}, t)] \\
& \frac{\partial d_{E}}{\partial r}(\bar{\rho}, t)=-\nu_{3}\left[z(t)+d_{E}(\bar{\rho}, t)\right]
\end{aligned}
$$

\section{APPENDIX II: MODIFIED MODEL}

The equations describing extracellular concentrations of conjugate, prodrug, and drug within the tumor were modified to include a convective current. Increased interstitial pressure acts to drive this convective velocity radially outward. The modified equations are

$$
\begin{gathered}
\frac{\partial C_{F}}{\partial t}+\nabla \cdot\left(c C_{F}\right)=D_{C} \Delta C_{F} \\
\quad-k_{a} C_{F}\left(A_{0}-C_{B}\right)+k_{d} C_{B} \\
\frac{\partial P}{\partial t}+\nabla \cdot(c P)=D_{P} \Delta P \\
\quad-\frac{V_{\max }\left(C_{F}+C_{B}\right) P}{K_{m}+P} \\
\frac{\partial D_{E}}{\partial t}+\nabla \cdot\left(c D_{E}\right)=D_{D} \Delta D_{E} \\
\quad+\frac{V_{\max }\left(C_{F}+C_{B}\right) P}{K_{m}+P}-a_{I} D_{E}
\end{gathered}
$$

where $c$ is the interstitial velocity. Baxter et al., 1989 derived the fluid velocity using Darcy's for flow through a porous medium. For axisymmetric flow, they write

$$
c=-K \frac{\partial p}{\partial r}
$$

where $K$ is the hydraulic conductivity of the interstitium and $p$ is the interstitial pressure. Combining this equation with a continuity equation for steady-state incompressible flow gives

$$
\Delta p=\frac{\alpha^{2}}{R}\left(p-p_{s s}\right)
$$

where $R$ is the tumor radius, $\alpha$ is a measure of the ratio of interstitial to vascular fluid flow, and $p_{s s}$ is the steady-state pressure. This equation is solved with no a no flux boundary condition at the center of the tumor and a matching condition at the tumor's edge $\left(p(R, t)=p_{\infty}\right)$. From this solution, the interstitial velocity can be computed as

$$
c(r)=\frac{K\left(p_{e}-p_{\infty}\right)}{r^{2}}\left[\frac{\alpha \cosh \alpha r / R-R \sinh \alpha r / R}{\sinh \alpha}\right]
$$

The convective velocity will differ in each region of the tumor and we use equation (18) to determine estimates for the velocity at different radial positions.

\section{Acknowledgments}

This work has been supported in part an NSF Graduate Fellowship (TLJ). The authors would like to thank Nathan Siemers, David Kerr, Vivekananda Vrudhula, and John Emsweiler for providing the experimental data.

\section{References}

Bagshawe, K. and Begent, R. (1996). First clinical experience with ADEPT. Adv. Drug Delivery Rev., 22, 365-367.

Baxter, L. and Jain, R. (1989). Transport of fluid and macromolecules in tumors. $i$. role of interstitial pressure and convection. Microvasc. Res, 37, 77-104.

Baxter, L. and Jain, R. (1991a). Transport of fluid and macromolecules in tumors. iii. role of binding and metabolism. Microvasc. Res., 41, 5-23.

Baxter, L. and Jain, R. (1991b). Transport of fluid and macromolecules in tumors. iv. a microscopic model of the perivascular distribution. Microvasc. Res., 41, 252-272.

Baxter, L. and Jain, R. (1996). Pharmacokinetics analysis of the microscopic distribution of enzyme-conjugated antibodies and prodrugs: comparison with experimental data. Br.J. Cancer, 73, 447-456.

Baxter, L., Yuan, F. and Jain, R. (1992). Pharmacokinetic analysis of the perivascular distribution of bifunctional antibodies 
and haptens: comparison with experimental data. Cancer Res. 52, 5838-5844.

Bosslet, K., Czech, J. and Hoffmann, D. (1994). Tumor-selective prodrug activation by fusion protein-mediated catalysis. Cancer Res, 54, 2151-2159.

Dalmark, M. and Storm, H. (1981). A fickian diffusion transport process with features of transport catalysis. Doxorubicin transport in red blood cells. J. Gen. Physiol. 78, 349-364.

DeGregorio, M., Lui, G., Macher, B. and Wilbur, J. (1984). Uptake, metabolism, and cytotoxicity of doxorubicin in human ewing's sarcoma and rhabdomyosarcoma cells. Cancer Chemother, Pharmacol., 12, 59-63.

Dordal, M., Ho, A., Jackson-Stone, M., Fu, Y., Goolsby, C. and Winter, J. (1995). Flow cytometric assesment of the cellular pharmacokinetics of fluorescent drugs. Cvtometry, $\mathbf{2 0}$, $307-314$

Dordal, M., Winter, J. and Atkinson, A. (1992). Kinetic analysis of $p$-glycoprotein-mediated doxorubicin efflux. J. Pharmacol. Exp. Ther., 263, 762-766.

Eccles, S., Court, W., Box, G. C. J. D., Melton, R. G. and Springer, C. (1994). Regression of established breast carcinoma xenografts with antibody-directed enzyme prodrug therapy against c-erb-b2 p185. Cancer Res, 54, $5171-5177$.

Endrich, B., Reinhold, H., Gross, J. and Intaglietta, M. (1979). Tissue perfusion inhomogeneity during early tumor growth in rats. J. Natl. Cancer Inst., 62, 387-395.

Gerlowski, L. and Jain, R. (1986). Microvascular permeability of normal and neoplastic tissues. Microvas. Res., 31, 288-305.

Jackson, T. L.. Lubkin, S. R. and Murray, J. D. (1999a). Theoretical analysis of conjugate localization in two-step cancer chemotherapy. Submitted.

Jackson, T. L., Lubkin, S. R., Siemers, N. O., Kerr, D. E., Senter, P. and Murray, J. D. (1999b). Mathematical and experimental analysis of localization of anti-tumor antibody enzyme conjugates. To Appear: Br. J. of Cancer.

Jain, R. (1987). Transport of macromolecules across tumor vasculature. Cancer Metastasis Rev., 6, 559-594.

Kerr, D., Schreiber, G., Vrudhula, B., Svensson, H., Hellstrom, K. and Senter, P. (1995). Regressions and cures of $m i$ noma xenografts following treatment with monoclonal an. sody $\beta$-lactamase conjugates in combination with anticancer prodrugs. Cancer Res., 55, 3558-3563.

Krogh, A. (1919). The number and distribution of capillaries in muscles with calculations of the oxygen pressure head necessary for supplying the tissue. $J$. of Physiol., 52, 409-415.

Melton, R. and Sherwood, R. (1996). Antibody-enzyme conjugates for cancer therapy. J. Natl. Cancer Inst., 88, 153-165.
Meyer, D., Jungheim L. Law, K. Mikolajczyk, S., Shepherd, T., Mackensen, D., Briggs, S. and Starling, J. (1993). Site-specific prodrug activation by antibody- $\beta$-lactamase conjugates: regression and long term growth inhibition of human colon carcinoma xenograft models. Cancer Res., 53, 3956-3963.

Niculescu-Duvaz, I. and Springer, C. (1996). Antibody-directed enzyme prodrug therapy (ADEPT): A targeting strategy in cancer chemotherapy. Current Medicinal Chemistry, 2, 687-706.

Nugent, L. and Jain, R. (1984). Extravascular diffusion in normal and neoplastic tissues. Cancer Res., 44, 238-244.

Paul, C., Peterson, C., Gahrton, G. and Lcokner, D. (1979). Uptake of free and dna-bound daunorubicin and doxorubicin into human leukemic cells. Cancer Chemother. Pharmacol., $2,49-52$.

Pietersz, G., Rowland, A., Smyth, M. and McKenzie, I. (1994). Chemoimmunoconjugates for the treatment of cancer. $A d v$. Immunol, 56, 301-387.

Robert, J., Illiadia, A. Hoent, B., Cano, J. P., Durand, M. and Lagarde, C. (1982). Pharmacokineties of adriamycin in patients with breast cancer: Correlation between pharmacokinetic parameters and clinical short-term reponse. Eur. J. Cancer Clin. Oncol., 18, 739-745.

Senter, P. and Svensson, H. (1997). A summary of monoclonal antibody-enzyme/prodrug combinations. Adv. Drug Delivery Rev., 22, 341-349.

Senter, P., Wallace, P., Svensson, H., Vrudhula, V., Kerr, D., Hellstrom, I. and Hellstrom, K. (1993). Generation of cytotoxic agents by targeted enzymes. Bioconjugate Chem. 4, 3-9.

Siemers, N., Kerr, D., Yarnold, S., Stebbins, M., Vrudhula, V., Hellstrom, L., Hellstrom, K. and Senter, P. (1997). L49-sfvB-Iactamase, a single chain anti-p97 antibody fusion protein. Bioconjugate Chem., 8, 510-519.

Springer, C., Bagshawe, K., Sharma, S., Searle, F., Boden, J., Antoniw, P., Burke, P., Rogers, G., Sherwood, R. and Melton, R. (1991). Abalation of human choriocarcinoma xenografts in nude mice by antibody-directed enzyme prodrug therapy (ADEPT) with three novel compounds. Eur. J. Cancer, 27, $1361-1366$.

Svensson, H., Vrudhula, V., Emswiler, J., MacMaster, J. Cosand, W., Wallace, P. and Senter, P. (1995). In vitro and in vivo activities of a doxorubicin prodrug in combination with monoclonal antibody B-lactamase conjugates. Cancer Res., 55 2357-2365.

Wallace, P., MacMaster, J., Smith, V., Kerr, D., Senter, P. and Cosand. W. (1994). Intratumoral generation of 5-fluorouracil mediated by an antibody-cytosine deaminase conjugate in combination with 5-fluorocytosine. Cancer Res., 54, 2719-2723. 


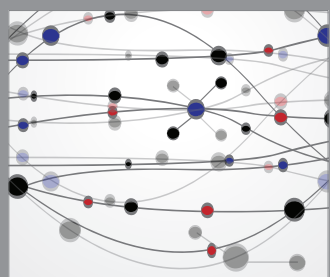

The Scientific World Journal
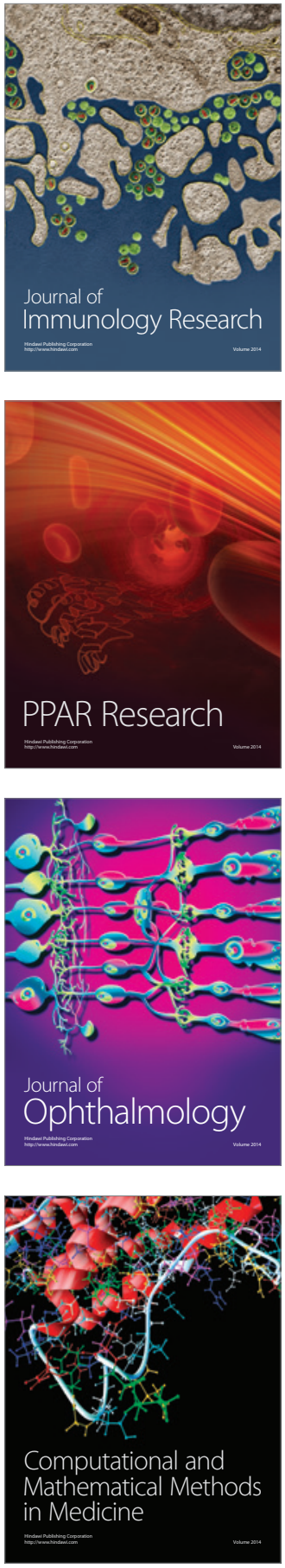

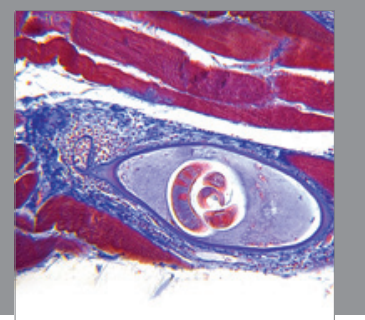

Gastroenterology

Research and Practice
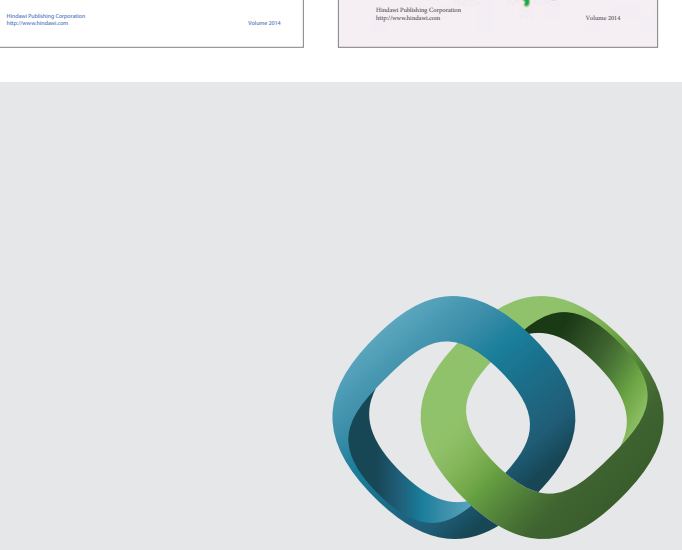

\section{Hindawi}

Submit your manuscripts at

http://www.hindawi.com
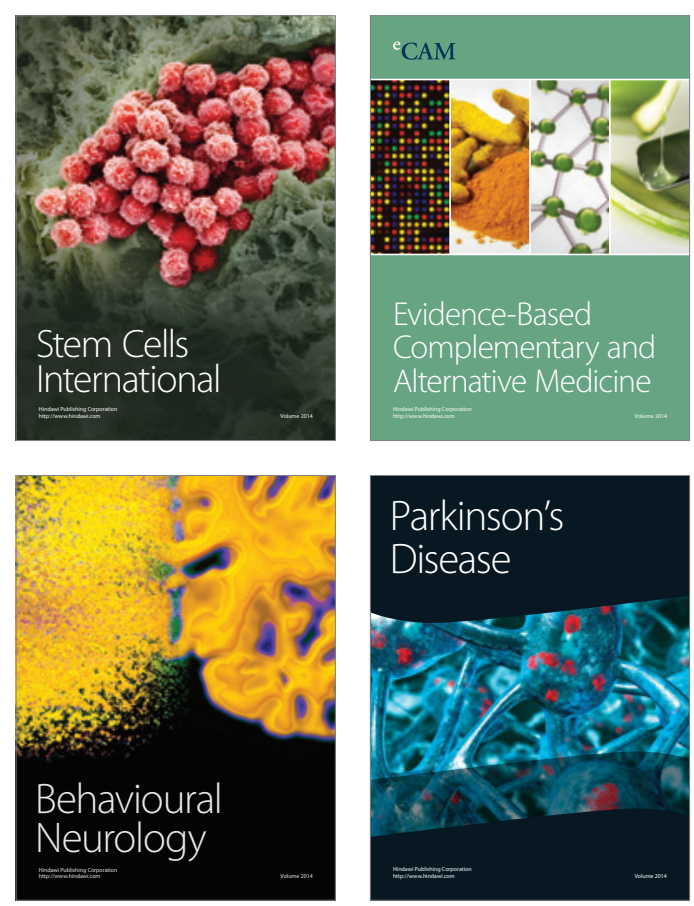

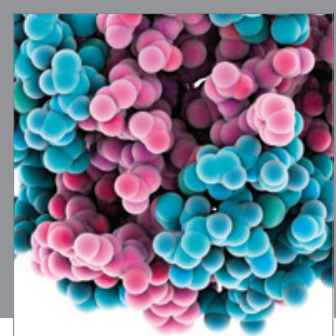

Journal of
Diabetes Research

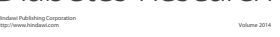

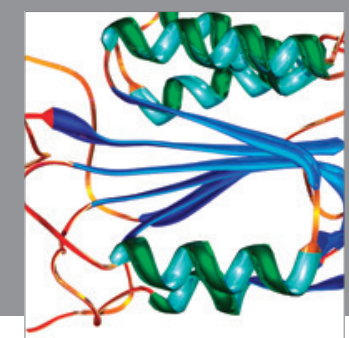

Disease Markers
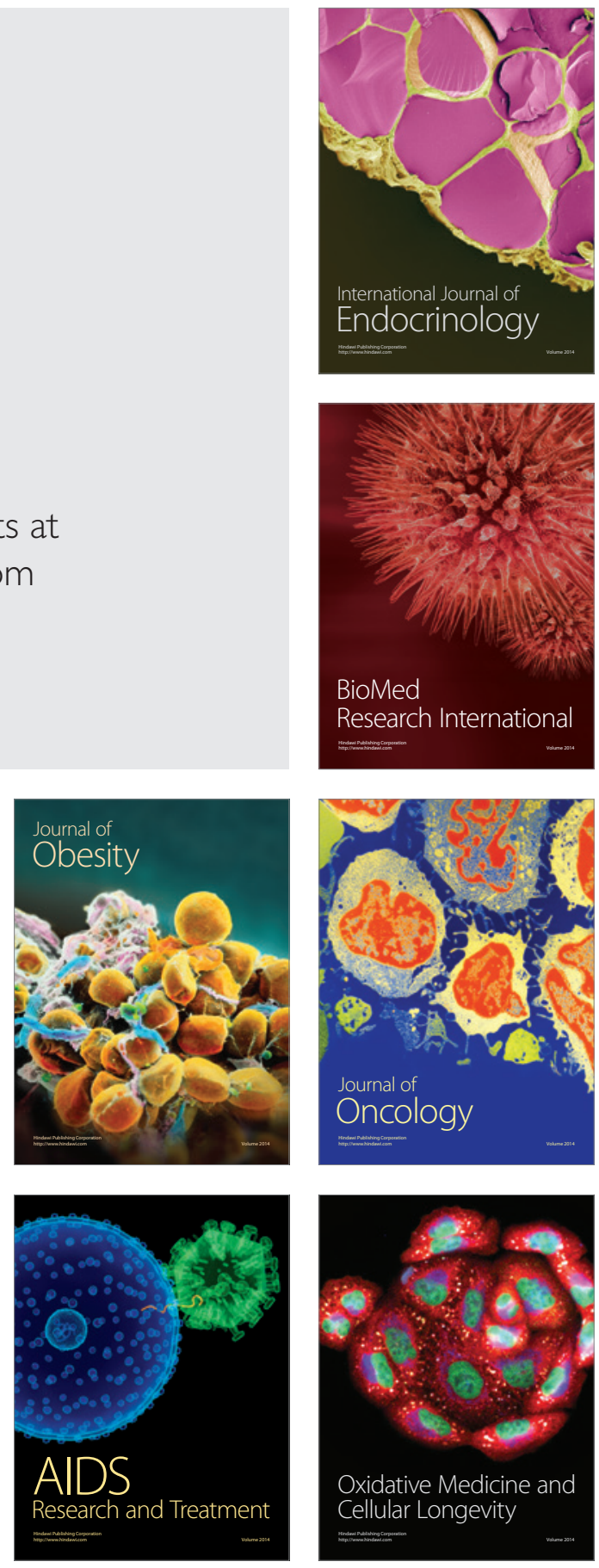